\title{
Unstable horizons and singularity development in holography
}

\author{
Pablo Bosch, ${ }^{a, d}$ Alex Buchel ${ }^{b, c, d}$ and Luis Lehner ${ }^{d}$ \\ ${ }^{a}$ Department of Physics 8 Astronomy and Guelph-Waterloo Physics Institute, \\ University of Waterloo, Waterloo, Ontario N2L 3G1, Canada \\ ${ }^{b}$ Department of Applied Mathematics, Ontario N6A 5B7, Canada \\ ${ }^{c}$ Department of Physics and Astronomy, University of Western Ontario, \\ Ontario N6A 5B\%, Canada \\ ${ }^{d}$ Perimeter Institute for Theoretical Physics, \\ Waterloo, Ontario N2J 2W9, Canada \\ E-mail: pbosch@perimeterinstitute.ca, abuchel@perimeterinstitute.ca, \\ llehner@perimeterinstitute.ca
}

ABSTRACT: In holographic applications one can encounter scenarios where a longwavelength instability can arise. In such situations, it is often the case that the dynamical end point of the instability is a new equilibrium phase with a nonlinear scalar hair condensate outside the black hole horizon. We here review holographic setups where symmetric horizons suffer from long-wavelength instabilities where a suitable equilibrium condensate phase does not exist. We study the dynamics of the simplest model in this exotic class, and show that it uncovers arbitrarily large curvatures in the vicinity of the horizon which asymptotically turn such region singular, at finite time with respect to the boundary theory.

KEYWORDS: Gauge-gravity correspondence, Spacetime Singularities

ARXIV EPRINT: 1704.05454 


\section{Contents}

1 Introduction 1

2 Exotic hairy black holes at equilibrium 3

3 Dynamics of the exotic unstable horizons $\quad 7$

3.1 Dynamical setup 8

$\begin{array}{ll}3.2 & \text { Dynamics of the symmetric sector }\end{array}$

3.3 Long-wavelength (GL-type) instability of the symmetric sector 11

$\begin{array}{ll}3.4 & \text { Fully non-linear evolutions of stable and unstable black holes } \\ \end{array}$

4 Conclusions $\quad 15$

$\begin{array}{lr}\text { A Numerical setup } & 19\end{array}$

$\begin{array}{lll}\text { A.1 Field redefinitions and the code equations } & 19\end{array}$

A.2 Apparent horizon and the boundary condition for $a \quad 22$

$\begin{array}{lll}\text { A.3 Initial conditions } & 23\end{array}$

A.4 Convergence tests 23

A.5 Event horizon finder 24

B Bounded scalar potentials in exotic holographic model 24

\section{Introduction}

The string theory/gauge theory correspondence $[1,2]$ is by now a mature framework exploited to address interesting questions in strongly coupled gauge theories that are often inaccessible with other theoretical tools. In a nutshell, this duality establishes a holographic correspondence (a dictionary) between two objects: a non-abelian gauge theory and a higher-dimensional gravitational theory/string theory in asymptotically anti de-Sitter space-time. One particularly appealing consequence of this correspondence is the fact that questions about the gauge theory in strongly coupled regimes are mapped onto questions in classical gravity. Likewise, a dual gauge theory perspective allows for different, and often intuitive, understanding of instabilities in black hole/black brane spacetimes.

Indeed, lets recall the physics of holographic superconductors [3, 4]. Consider the four-dimensional effective gravitational action ${ }^{1}$ in asymptotically $A d S_{4}$ (dual to a threedimensional conformal field theory $C F T_{3}$ ),

$$
S_{4}=\frac{1}{2 \kappa^{2}} \int_{\mathcal{M}_{4}} d x^{4}\left[R+6-\frac{1}{4} F^{\mu \nu} F_{\mu \nu}-\frac{1}{2}(\nabla \phi)^{2}+\phi^{2}\right] .
$$

\footnotetext{
${ }^{1}$ We set the radius $L$ of an asymptotic $A d S_{4}$ geometry to unity.
} 
The four dimensional gravitational constant $\kappa$ is related to a central charge $c$ of the $C F T_{3}$ as

$$
c=\frac{192}{\kappa^{2}},
$$

$F_{\mu \nu}$ is a field strength of a global $\mathrm{U}(1)$ symmetry of the CFT, and $\phi$ is a (neutral) gravitational bulk scalar with

$$
L^{2} m_{\phi}^{2}=-2,
$$

which is dual to a dimension $\Delta_{\phi}=2$ operator $\mathcal{O}_{\phi}$ of a boundary theory. ${ }^{2}$ Note that there is $\mathbb{Z}_{2}$ symmetry in the model, associated with this scalar, $\phi \leftrightarrow-\phi$. As it is well-known, there are two phases of equilibrium states of this $C_{F} T_{3}$ at a finite temperature $T$ and a U(1) global symmetry chemical potential $\mu$, distinguished whether $\left\langle\mathcal{O}_{\phi}\right\rangle=0$ or $\left\langle\mathcal{O}_{\phi}\right\rangle \neq 0$. The $\left\langle\mathcal{O}_{\phi}\right\rangle=0$ phase exists for arbitrary temperature $T \geq 0$ and it is gravitationally described by Reissner-Nordstrom $A d S_{4}$ black brane with unbroken $\mathbb{Z}_{2}$ symmetry, correspondingly $\phi \equiv 0$. For sufficiently small $T / \mu$ this $\mathbb{Z}_{2}$ symmetric phase becomes unstable [4]: on the gauge theory side of the correspondence the instability is a generic instability of the order parameter in the mean-field theory of thermal second-order phase transitions; on the gravity side, this is a Gregory-Laflamme (GL) type instability [6] (in the sense of being unstable to long-wavelength perturbations) due to scalarization of the Reissner-Nordstrom $A d S_{4}$ black brane horizon. To understand the gravitational origin of the instability the authors of [4] noted that even though the scalar $\phi$ is above the $A d S_{4}$ Breitenlohner-Freedman (BF) bound

$$
m_{\phi}^{2}=-2 \quad>\quad m_{\mathrm{BF}\left[\mathrm{AdS}_{4}\right]}^{2}=-\frac{(4-1)^{2}}{4 L^{2}}=-\frac{9}{4},
$$

as the Reissner-Nordstrom $A d S_{4}$ black brane becomes extremal $(T / \mu \rightarrow 0)$, it develops $A d S_{2} \times R^{2}$ near horizon geometry with the curvature radius $L_{2}^{2}=\frac{L^{2}}{6}$. In this limit

$$
m_{\phi}^{2}=-2 \quad<\quad m_{\mathrm{BF}\left[\mathrm{AdS}_{2}\right]}^{2}=-\frac{(2-1)^{2}}{4 L_{2}^{2}}=-\frac{3}{2},
$$

and the bulk scalar $\phi$ becomes unstable (the quasinormal frequency of its linearized fluctuations has $\operatorname{Im}[\omega]>0)$. The condensation of the gravitational scalar $\phi$ at low temperatures is dynamically saturated by nonlinear effects, spontaneously breaking $\mathbb{Z}_{2}$ symmetry and leading to a new equilibrium phase of the CFT with $\left\langle\mathcal{O}_{\phi}\right\rangle \neq 0$.

There exist many studies and generalizations of the described phenomena in holography. ${ }^{3}$ In this paper we focus on a less-known, exotic property of certain black brane/black hole horizons. As in the example of the holographic superconductor above, imagine a holographic ${ }^{4}$ horizon with a discrete (or continuous) symmetry. Suppose that there is a critical energy ${ }^{5}$ or energy density (for gauge theory states with translational invariance) below which the horizon becomes unstable with respect to symmetry breaking (GL) fluctuations. There is an equilibrium phase with spontaneously broken symmetry, branching

\footnotetext{
${ }^{2} \phi$ has two alternative quantizations in $A d S_{4}$ [5]; our results do not depend on this choice.

${ }^{3}$ See $[7]$ and references therein.

${ }^{4}$ We point out this feature occurs in top down holographic models, and thus is of importance to issues of equilibration and thermalization in strongly coupled gauge theories.

${ }^{5}$ As we study dynamical phenomena, we work in a microcanonical ensemble.
} 
off the GL onset of the instability, yet, this phase does not exist below the critical energy; moreover, it has lower entropy above the criticality than the symmetric phase. Thus, the horizon representing the symmetric thermal state is unstable, but it is unknown what the end point of its instability is.

To our knowledge, the first realization of the above exotic scenario appeared in [8] which was later found in a top-down holographic model in [9]. Finally, the same exotic physics is behind the leading instability of small black holes in $A d S_{5} \times S^{5}$ (dual to $\mathrm{SO}(6$ )symmetric states of strongly coupled $\mathcal{N}=4$ SYM plasma) [10-13]. Here, we study the endpoint of this exotic horizon instability.

In the next section we briefly review the bottom-up model of [8]. We discuss the equilibrium states of the system, and the linearized instability of symmetric phase states at low energy densities. We construct the symmetry-broken phase of the system and demonstrate that it is never preferred dynamically. In section 3 we employ a characteristic formulation of the gravitational dynamics [14] in our exotic model. We confirm the onset of the GL instability dynamically, and compare the linear growth (below the criticality) and decay (above the criticality) rates of the symmetry breaking fluctuations with the corresponding quasinormal mode (QNM) computations of section 2. Next, we present results for the full-nonlinear evolutions of unstable horizons. Details of the numerical implementation as well as the convergence and the validation of the code are delegated to appendix A. We conclude and discuss open questions in section 4 .

\section{Exotic hairy black holes at equilibrium}

In this section we review the bottom-up holographic model of the exotic black holes presented in [8].

The effective four-dimensional gravitational bulk action, dual to a field-theoretic setup discussed in the introduction, takes the form

$$
\begin{aligned}
S_{4} & =S_{\mathrm{CFT}}+S_{r}+S_{i}=\frac{1}{2 \kappa^{2}} \int d x^{4} \sqrt{-\gamma}\left[\mathcal{L}_{\mathrm{CFT}}+\mathcal{L}_{r}+\mathcal{L}_{i}\right], \\
\mathcal{L}_{\mathrm{CFT}} & =R+6, \quad \mathcal{L}_{r}=-\frac{1}{2}(\nabla \phi)^{2}+\phi^{2}, \quad \mathcal{L}_{i}=-\frac{1}{2}(\nabla \chi)^{2}-2 \chi^{2}-g \phi^{2} \chi^{2} ;
\end{aligned}
$$

where we split the action into (a holographic dual to) a CFT part $S_{\mathrm{CFT}}$; its deformation by a relevant operator $\mathcal{O}_{r}$; and a sector $S_{i}$ involving an irrelevant operator $\mathcal{O}_{i}$ along with its mixing with $\mathcal{O}_{r}$ under the renormalization-group dynamics. We take bulk quantization so that the scaling dimension of $\mathcal{O}_{r}$ is $\Delta_{r}=2$; the scaling dimension of $\mathcal{O}_{i}$ is $\Delta_{i}=4$. In order to have asymptotically $A d S_{4}$ solutions, we assume that only the normalizable mode of $\mathcal{O}_{i}$ is nonzero near the boundary.

The gravitational action (2.1) has $\mathbb{Z}_{2} \times \mathbb{Z}_{2}$ discrete symmetry that acts as a parity transformation on the scalar fields $\phi$ and $\chi$. The discrete symmetry $\phi \leftrightarrow-\phi$ is explicitly broken by the relevant deformation of the CFT,

$$
\mathcal{H}_{\mathrm{CFT}} \rightarrow \mathcal{H}_{\mathrm{CFT}}+\Lambda \mathcal{O}_{r},
$$



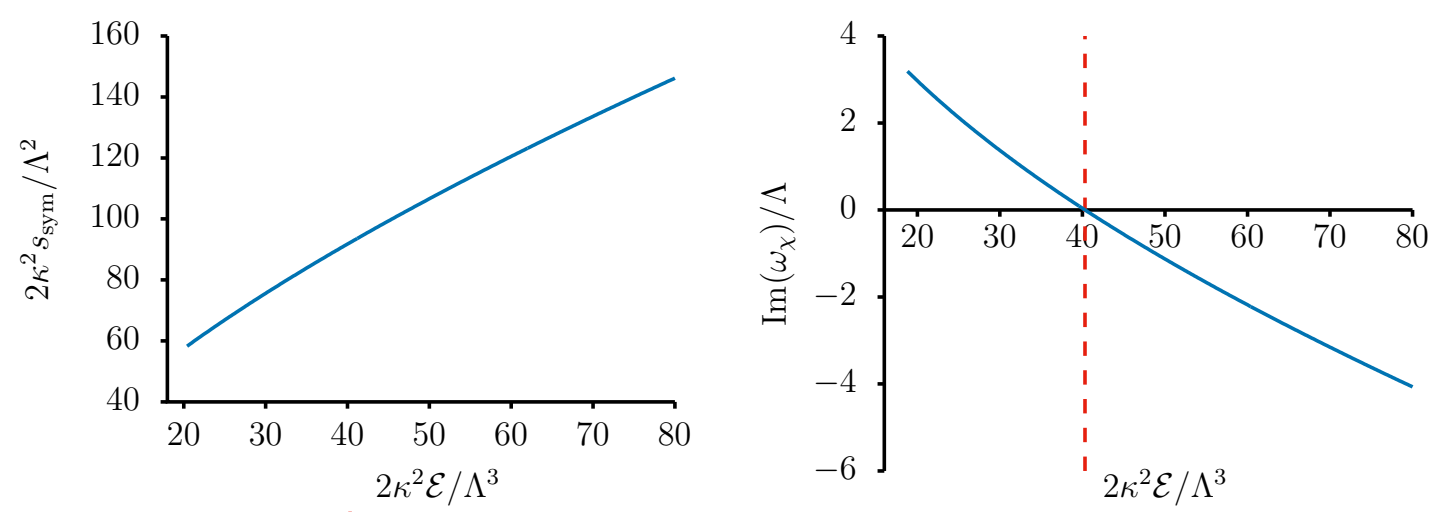

Figure 1. Entropy density $s_{\text {sym }}$ of the $\mathbb{Z}_{2}$-symmetric phase, i.e., with $\left\langle\mathcal{O}_{i}\right\rangle=0$, of exotic black holes as a function of energy density $\mathcal{E}$ (left panel). As the energy density is decreased below the critical one $\mathcal{E}_{\text {crit }}$, denoted by a vertical (red) dashed line, and given that the symmetric phase is perturbatively unstable with respect to linearized $\mathbb{Z}_{2}$-symmetry breaking fluctuations, the imaginary part of the frequency $\omega_{\chi}$ of these fluctuations at zero spatial momenta is positive (right panel).

with $\Lambda$ being the deformation mass scale, while the $\chi \leftrightarrow-\chi$ symmetry is broken spontaneously. The mechanism for the long-wavelength instability at play in (2.1) was motivated through the following observations $[8,15]$ :

- consider the linearized dynamics of the $\chi$-sector in the mass-deformed CFT dual to $S_{\mathrm{CFT}}+S_{r}$ in $(2.1)$

- for the quartic coupling $g<0$, the scalar $\chi$ has an effective mass

$$
m_{\chi}^{2}=4-2|g| \phi^{2}
$$

- homogeneous and isotropic thermal equilibrium states of $S_{\mathrm{CFT}}+S_{r}$ at low temperature (energy densities) would result in large values of $\phi$ at the horizon of the dual gravitational description, thus driving $m_{\chi}^{2}$ below the effective BF bound. ${ }^{6}$

A detailed analysis of the homogeneous and equilibrium states of the holographic model (2.1) in the canonical ensemble were presented in [8]. Here, we present results in the microcanonical ensemble. We omit all the technical details as the following discussion is a special case of the dynamical setup of section 3 .

- There are two equilibrium phases of the holographic model (2.1), distinguished by the symmetry property under $\chi \leftrightarrow-\chi$ : the symmetric phase with $\left\langle\mathcal{O}_{i}\right\rangle=0$, and the symmetry broken phase with $\left\langle\mathcal{O}_{i}\right\rangle \neq 0$.

- The entropy density of the symmetric phase $s_{\text {sym }}$ as a function of the energy density $\mathcal{E}$ is presented in figure 1 . While this phase is thermodynamically stable $\frac{\partial^{2} \mathcal{E}}{\partial s_{\mathrm{sym}}^{2}}>0$,

\footnotetext{
${ }^{6}$ Here we take 'BF bound' in regards to original $A d S_{4}$ geometry, neglecting the backreaction of $\phi$ scalar. This is nothing but a motivation for engineering a model (2.1) with a horizon instability. The instability was explicitly confirmed with direct computations in [16].
} 

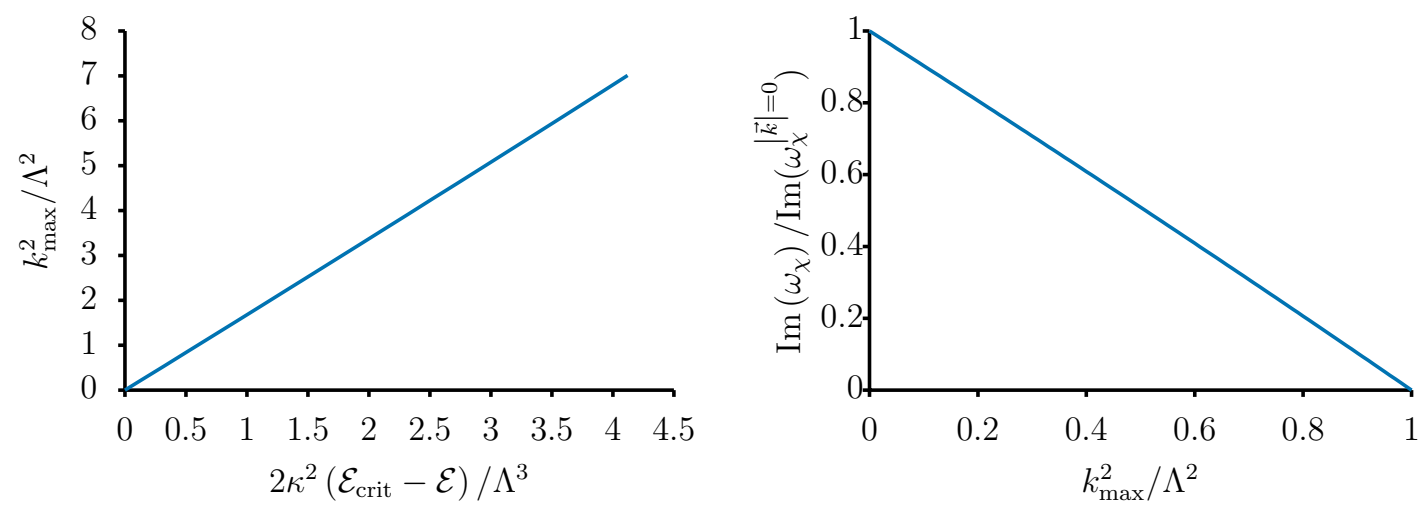

Figure 2. When $\mathcal{E}<\mathcal{E}_{\text {crit }}, \mathbb{Z}_{2}$-symmetry breaking fluctuations in the symmetric phase of the exotic black holes are unstable; the instability persists for the range of the spatial momenta (along the translationally invariant directions of the horizon) $\vec{k}$ of the fluctuations, $|\vec{k}| \in\left[0, k_{\max }\right]$. Right panel shows a characteristic dependence of $\operatorname{Im}\left(\omega_{\chi}\right)$ on $|\vec{k}|\left(\right.$ here $\mathcal{E} / \mathcal{E}_{\text {crit }}=0.89780(8)$ ).

it is perturbatively unstable with respect to a linearized symmetry breaking fluctuations [16]: for $\mathcal{E}<\mathcal{E}_{\text {crit }}$, with

$$
\frac{2 \kappa^{2} \mathcal{E}_{\text {crit }}}{\Lambda^{3}}=40.320(4),
$$

the quasinormal modes of the symmetry breaking linearized $\chi$-fluctuations develop a positive imaginary part, $\operatorname{Im}\left(\omega_{\chi}\right)>0$. As emphasized in [16], this model is one of the explicit counterexamples of the Gubser-Mitra "correlated stability conjecture" [17, 18].

- Notice that there is a relation between the behavior of the unstable $\chi$-mode and the ' $G L$ ' instability, in that the $\chi$-instability requires long wavelength modes, i.e., the instability is cut-off at

$$
|\vec{k}| \leq k_{\max } \propto\left(\mathcal{E}_{\text {crit }}-\mathcal{E}\right)^{1 / 2} .
$$

See figure 2 for further details.

However, there is a clear distinction: in the GL instability [6] the unstable mode is hydrodynamic, while the $\chi$-QNM behaves non-hydrodynamically away from the critical point, i.e., $\operatorname{Im}\left(\omega_{\chi}\right) \neq 0$ as the spatial momentum (along the translationally invariant directions of the horizon) vanishes, $|\vec{k}|=0$.

- The expectation value of $\left\langle\mathcal{O}_{i}\right\rangle \neq 0$ in the symmetry broken phase of the model as a function of the equilibrium energy density is presented in figure 3. This phase exists only for $\mathcal{E}>\mathcal{E}_{\text {crit }}$, with

$$
\lim _{\mathcal{E} \rightarrow \mathcal{E}_{\text {crit }}+}\left(\left\langle\mathcal{O}_{i}\right\rangle \propto-\left(\mathcal{E}-\mathcal{E}_{\text {crit }}\right)^{1 / 2}\right)=0 .
$$

The equilibrium symmetry broken phase is never realized in a microcanonical ensemble as it has smaller entropy density compared to the symmetric phase for the same energy density. 

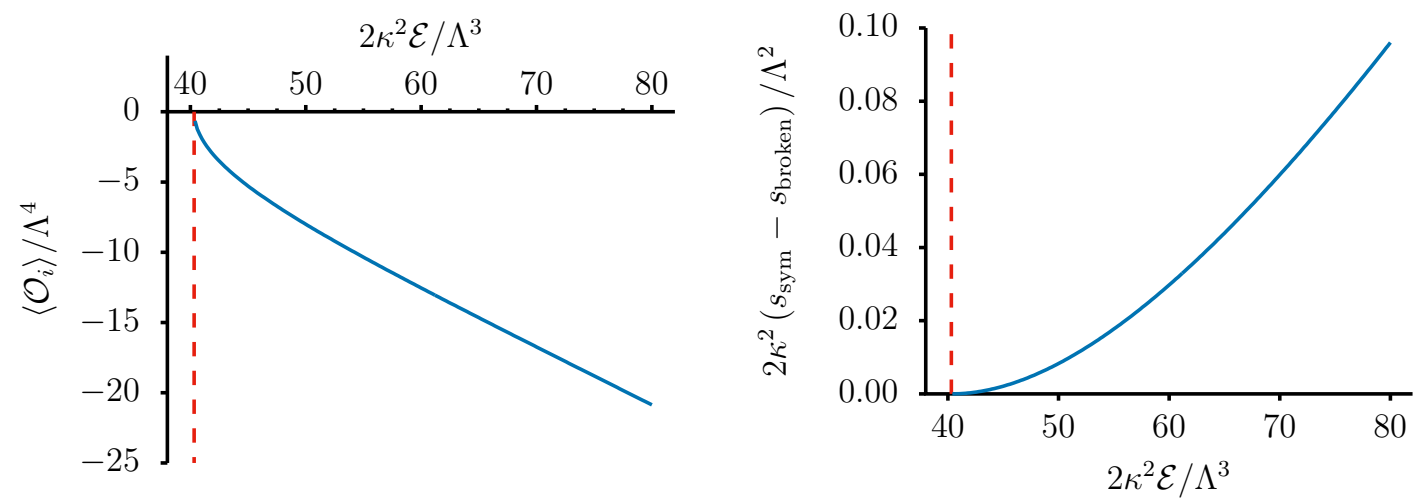

Figure 3. Exotic black holes have a new equilibrium phase with spontaneously broken $\mathbb{Z}_{2}$-symmetry at energy densities exceeding the critical one, denoted by a vertical (red) dashed line. This phase is characterized by $\left\langle\mathcal{O}_{i}\right\rangle \neq 0$, with the expectation value vanishing precisely at $\mathcal{E}=\mathcal{E}_{\text {crit }}$ (left panel). The equilibrium symmetry breaking phase $\left\langle\mathcal{O}_{i}\right\rangle \neq 0$ is never realized in a microcanonical ensemble as its entropy density is always below the corresponding entropy density of the symmetric phase (right panel).
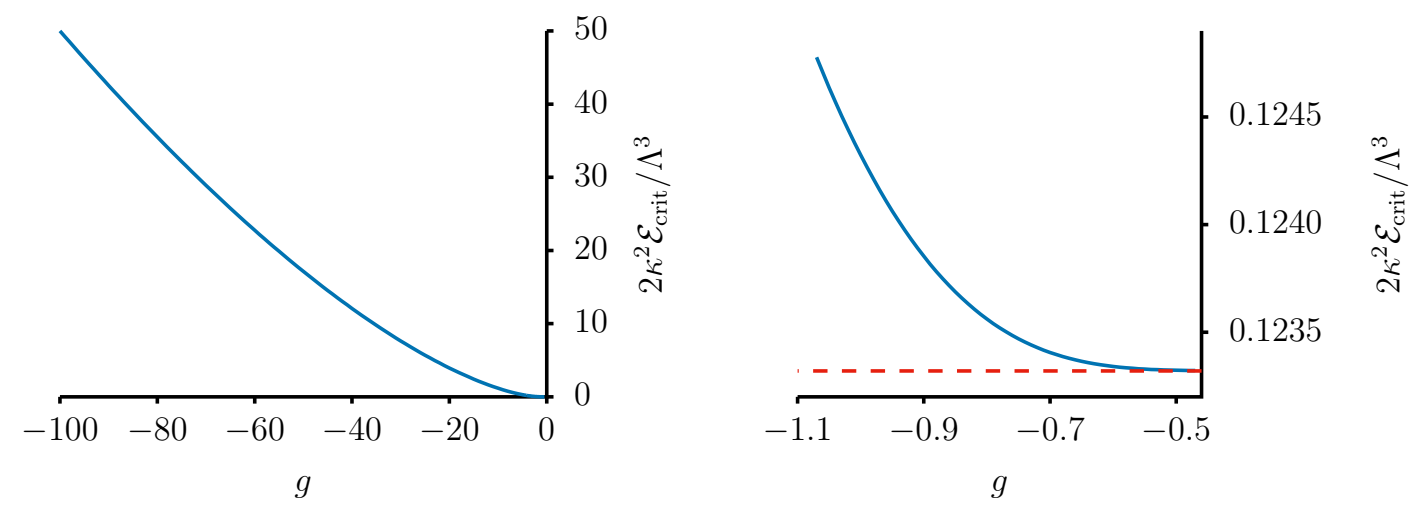

Figure 4. Critical energy density of the leading instability of the symmetric phase as a function of the nonlinear coupling $g$. It appears that the instability persists in the limit $g \rightarrow 0_{-}$(right panel). The (red) dashed line identifies the vacuum energy of the symmetric phase, see (2.9).

- figure 1 exhibits the leading instability at low-energies of the symmetric phase in the holographic model (2.1). In fact, there is a tower of unstable modes (overtones) with critical energies $\mathcal{E}_{\text {crit }}^{(n)}$,

$$
\mathcal{E}_{\text {crit }}^{(n)}<\mathcal{E}_{\text {crit }}^{(n-1)}, \quad \mathcal{E}_{\text {crit }}^{(0)} \equiv \mathcal{E}_{\text {crit }}, \quad \mathcal{E}_{\text {crit }}^{(1)} \approx 0.26380(6) \mathcal{E}_{\text {crit }},
$$

parameterized by the number of nodes $(n)$ in the radial profile of the linearized gravitation fluctuations $\chi$. Each subleading instability of the symmetric phase identifies a branch point of a new unstable phase with $\left\langle\mathcal{O}_{i}\right\rangle \neq 0$. Properties of these new phases are analogous to the broken phase in figure 3 , see also [8].

- The analysis reported above was performed with the nonlinear coupling in the effective action (2.1) set to $g=-100$. The phase diagram of the model does not change 

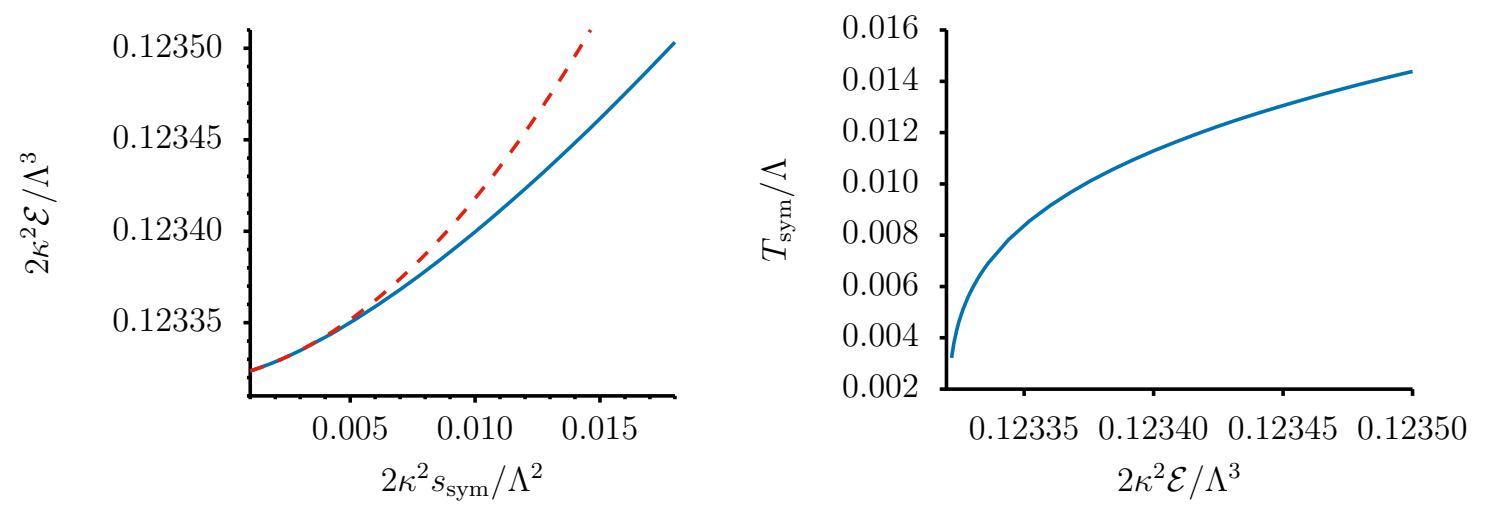

Figure 5. The left panel shows the energy density of the symmetric phase as a function of the entropy density. The (red) dashed line is the extrapolation of the energy-entropy data (solid blue line) in the limit $s_{\mathrm{sym}} \rightarrow 0$. The extrapolation is used to estimate the vacuum energy of the symmetric phase (2.9). The right panel shows the dependence of the symmetric phase black hole temperature as a function of the energy density.

as $g$ changes, as long as $g<0$, see figure 4 . The (red) dashed line in the right panel represents the estimate for the vacuum energy of the symmetric phase:

$$
\frac{2 \kappa^{2} \mathcal{E}^{\text {vacuum }}}{\Lambda^{3}}=0.1233(2)
$$

Notice that $\mathcal{E}_{\mathrm{sym}}^{\text {vacuum }} \rightarrow 0$ in the conformal limit $\Lambda \rightarrow 0$; to obtain the better estimate for $\mathcal{E}^{\text {vacuum }}$ we extended the analysis of the symmetric phase to the low-entropy region, as shown in figure 5, and extrapolated the energy-entropy data to zero entropy density (indicated by the (red) dashed line). The right panel shows the dependence of the temperature $T_{\text {sym }}$ of the symmetric phase black hole - the limit $\mathcal{E} \rightarrow \mathcal{E}^{\text {vacuum }}$ appears to correspond to an extremal limit.

In this section we focused on the static phase diagram, along with the linearized (in)stabilities of these phases, of the holographic action (2.1), dual to non-conformal $Q F T_{3}$ in Minkowski space-time $R^{1,2}$. In what follows we will discuss the dynamical case. In section 4, we comment on properties of the model with $Q F T_{3}$ residing in $R \times S^{2}$. Additionally, we comment on the extension of the model (2.1) with the gravitational potential for the scalar $\chi$ bounded from below.

\section{Dynamics of the exotic unstable horizons}

In this section we discuss dynamical properties of the holographic model (2.1), with the boundary $Q F T_{3}$ formulated in $R^{1,2}$. We follow closely the holographic numerical framework in the characteristic formulation as described in, e.g., [14]. 


\subsection{Dynamical setup}

We assume translational invariance along the spatial directions of the boundary. The relevant fields are described by

$$
\begin{aligned}
d s_{4}^{2} & =2 d t(d r-A(t, r) d t)+\Sigma(t, r)^{2}\left[d x_{1}^{2}+d x_{2}^{2}\right], \\
\phi & =\phi(t, r), \quad \chi=\chi(t, r) .
\end{aligned}
$$

Einstein equations define the following evolution equations of motion:

$$
\begin{aligned}
& 0=d_{+}^{\prime} \Sigma+d_{+} \Sigma(\ln \Sigma)^{\prime}-\frac{3}{2} \Sigma-\frac{1}{4} \Sigma\left(\phi^{2}-2 \chi^{2}-g \phi^{2} \chi^{2}\right), \\
& 0=d_{+}^{\prime} \phi+d_{+} \phi(\ln \Sigma)^{\prime}+\frac{d_{+} \Sigma}{\Sigma} \phi^{\prime}+\phi\left(1-g \chi^{2}\right), \\
& 0=d_{+}^{\prime} \chi+d_{+} \chi(\ln \Sigma)^{\prime}+\frac{d_{+} \Sigma}{\Sigma} \chi^{\prime}-\chi\left(2+g \phi^{2}\right), \\
& 0=A^{\prime \prime}-2 \frac{d_{+} \Sigma}{\Sigma^{2}} \Sigma^{\prime}+\frac{1}{2} d_{+} \phi \phi^{\prime}+\frac{1}{2} d_{+} \chi \chi^{\prime},
\end{aligned}
$$

together with the constraint equations:

$$
\begin{aligned}
0= & \Sigma^{\prime \prime}+\frac{1}{4} \Sigma\left(\left(\phi^{\prime}\right)^{2}+\left(\chi^{\prime}\right)^{2}\right) \\
0= & d_{+}^{2} \Sigma-2 A d_{+}^{\prime} \Sigma-\frac{d_{+} \Sigma}{\Sigma^{2}}\left(A \Sigma^{2}\right)^{\prime} \\
& +\frac{1}{4} \Sigma\left(\left(d_{+} \phi\right)^{2}+\left(d_{+} \chi\right)^{2}+2 A\left(6+\phi^{2}-2 \chi^{2}-g \phi^{2} \chi^{2}\right)\right),
\end{aligned}
$$

where $^{\prime} \equiv \partial_{r}$ and $d_{+} \equiv \partial_{t}+A \partial_{r}$. The constraint equations are preserved by the evolution equations provided they are satisfied at a given timelike surface (e.g., [19-21]) — which in our case is the AdS boundary.

The general asymptotic boundary $(r \rightarrow \infty)$ solution of the equations of motion, given by

$$
\begin{aligned}
\Sigma= & r+\lambda(t)-\frac{1}{8} p_{1}^{2} \frac{1}{r}+\mathcal{O}\left(\frac{1}{r^{2}}\right), \\
A= & \frac{r^{2}}{2}+\lambda(t) r-\frac{1}{8} p_{1}^{2}+\frac{1}{2} \lambda(t)^{2}-\dot{\lambda}(t) \\
& +\left(\mu-\frac{1}{4} p_{1} p_{2}(t)-\frac{1}{4} p_{1}^{2} \lambda(t)\right) \frac{1}{r}+\mathcal{O}\left(\frac{1}{r^{2}}\right), \\
\phi= & \frac{p_{1}}{r}+\frac{p_{2}(t)}{r^{2}}+\mathcal{O}\left(\frac{1}{r^{3}}\right), \\
\chi= & \frac{q_{4}(t)}{r^{4}}+\mathcal{O}\left(\frac{1}{r^{5}}\right),
\end{aligned}
$$

is characterized by two constants $\left\{p_{1}, \mu\right\}$, and three dynamical variables $\left\{p_{2}(t), q_{4}(t), \lambda(t)\right\}$. These parameters have the following interpretation: 
- $p_{1}$ and $p_{2}(t)$ are correspondingly the non-normalizable and normalizable coefficients of the bulk scalar $\phi$, identified with the deformation mass scale $\Lambda$ and the expectation value of the relevant operator $\mathcal{O}_{r}$ of the dual $Q F T_{3}$,

$$
p_{1}=\Lambda, \quad p_{2}(t)=\left\langle\mathcal{O}_{r}(t)\right\rangle
$$

- $q_{4}(t)$ is the normalizable coefficient of the bulk scalar $\chi$, identified with the expectation value of the $\mathbb{Z}_{2}$-symmetry breaking irrelevant operator $\mathcal{O}_{i}$ of the dual $Q F T_{3}$,

$$
q_{4}(t)=\left\langle\mathcal{O}_{i}(t)\right\rangle
$$

- $\mu$ is related to the conserved energy density $\mathcal{E}$ of the boundary $Q F T_{3}$ as follows

$$
\frac{2 \kappa^{2} \mathcal{E}}{\Lambda^{3}}=\frac{-4 \mu}{\Lambda^{3}}
$$

- $\lambda(t)$ is the residual radial coordinate diffeomorphisms parameter

$$
r \rightarrow r+\lambda(t)
$$

which can adjusted to keep the apparent horizon at a fixed location, which in our case will be $r=1$ :

$$
\left.\left(\partial_{t}+A(t, r) \partial_{r}\right) \Sigma(t, r) \equiv d_{+} \Sigma(t, r)\right|_{r=1}=0 .
$$

To initialize evolution at $t=0$, we provide the bulk scalar profiles,

$$
\phi(t=0, r)=\frac{p_{1}}{r}+\mathcal{O}\left(\frac{1}{r^{2}}\right), \quad \chi(t=0, r)=\mathcal{O}\left(\frac{1}{r^{4}}\right)
$$

along with the values of $\left\{p_{1}, \mu\right\}$, specifying the dual $Q F T_{3}$ mass scale $\Lambda$ (3.6) and the initial state energy density $\mathcal{E}(3.8)$. The constraint equation (3.3) is then used to determine an initial profile $\Sigma(t=0, r)$. Eqs. (3.2) are then employed to evolve such data (3.11) in time. The second constraint (3.4), representing the conservation of the energy density, is enforced requiring that a parameter $\mu$ in the asymptotic expansion of $A$, see (3.5), is time-independent.

Details of the numerical implementation, specific choices of the initial conditions (3.11) used, and code convergence tests can be found in appendix A.

\subsection{Dynamics of the symmetric sector}

To study dynamics in the symmetric sector, we adopt initial conditions as described in appendix A.3 with $\mathcal{A}_{p} \neq 0$ and $\mathcal{A}_{q}=0$, implying that (in $\lambda_{0} \equiv 0$ gauge)

$$
\left.\phi\right|_{t=0}=\frac{p_{1}}{r}+\mathcal{A}_{p} \frac{\exp \left(-\frac{1}{r}\right)}{r^{2}}, \quad \chi(t, x) \equiv 0 .
$$



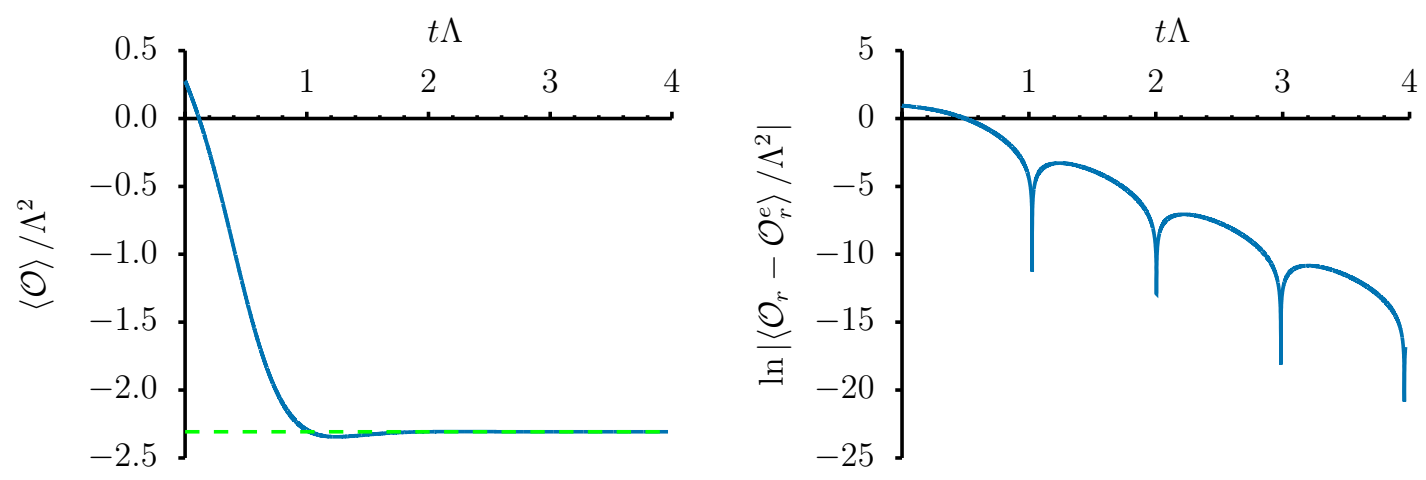

Figure 6. Time evolution of a typical state in $\mathbb{Z}_{2}$-symmetric phase of exotic black holes. The (green) dashed line is the equilibrium value $\left\langle\mathcal{O}_{r}^{e}\right\rangle$ of the operator $\mathcal{O}_{r}$. Right panel shows that approach towards equilibrium occurs in characteristic quasinormal mode ringing of the black hole horizon.
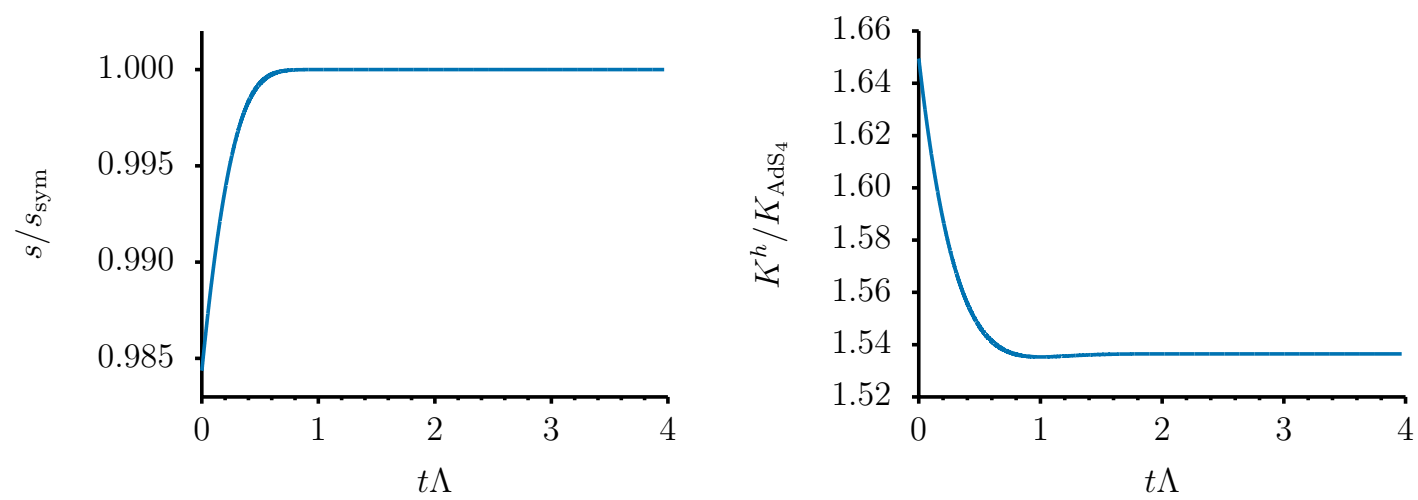

Figure 7. Dynamical entropy density $s$ relative to the equilibrium entropy density $s_{\text {sym }}$ in $\mathbb{Z}_{2}$-symmetric sector of exotic black holes (left panel). Corresponding evolution of the bulk Kretschmann scalar (right panel).

Results of a typical evolution are presented in figures 6 and 7 . Here, the energy density is below the critical one (2.5),

$$
\mathcal{E}=0.793642 \mathcal{E}_{\text {crit }} \quad \Longleftrightarrow \quad \mu=-4 \Lambda^{3} .
$$

The left panel of figure 6 shows time evolution of the expectation value of $\mathcal{O}_{r}$. Within a time scale $t \sim \Lambda^{-1}$ the system equilibrates. The equilibrium expectation value, defined as

$$
\left\langle\mathcal{O}_{r}^{e}\right\rangle=\lim _{t \Lambda \rightarrow \infty}\left\langle\mathcal{O}_{r}(t)\right\rangle,
$$

is represented by a (green) dashed line. We used the value of $\left\langle\mathcal{O}_{r}^{e}\right\rangle$ obtained from the independent analysis of the static configurations, reported in section 2, evaluated at the energy density (3.13). Consistency of (3.14) is an important check of our evolution. The right panel of figure 6 illustrates the system's approach to equilibrium, which displays a typical $\phi$ quasinormal mode ring-down of the exotic black hole horizon.

The entropy density is an intrinsically equilibrium concept in QFTs. One benefit of the holographic framework is that it provides a well-motivated notion of the non-equilibrium 
(even far from equilibrium) entropy. Following [22, 23] we identify nonequilibrium entropy density $s$ with the Bekenstein-Hawking entropy corresponding to the apparent horizon (see (3.10)) area density

$$
s(t)=\left.\frac{2 \pi}{\kappa^{2}} \Sigma(t, r)^{2}\right|_{r=1} .
$$

The right panel of figure 7 shows the evolution of thus defined dynamical entropy density. Notice that in line with the second law of thermodynamics, $\dot{s}(t) \geq 0$ and approaches at late times the equilibrium value $s_{\mathrm{sym}}$, computed independently for the static configuration with the energy density (3.13).

While we can study the non-equilibrium dynamics in $\mathbb{Z}_{2}$-symmetric sector of the holographic model (2.1), completely suppressing the $\chi$-scalar fluctuations as in (3.12), at $\mathcal{E}<\mathcal{E}_{\text {crit }}$, and in particular in the discussed example (3.13), this is an unphysical approximation - in realistic settings the fluctuations of the $\chi$ scalar will always be present, and would destabilize this $\mathbb{Z}_{2}$-symmetric dynamics. In the right panel of figure 7 we show the time dependence of the bulk Kretschmann scalar $K$ evaluated at the apparent horizon,

$$
K(t)=\left.R_{a b c d} R^{a b c d}\right|_{(t, r=1)},
$$

relative to the $A d S_{4}$ Kretschmann scalar $K_{A d S_{4}}$ (recall $K_{A d S_{4}}=$ const $=24$ ), to emphasize the fact that even if the symmetric sector is unstable, its bulk dynamics is weakly curved. Thus, higher derivative supergravity and string corrections are arguably irrelevant for the onset of the $\mathbb{Z}_{2}$ symmetry breaking instability of the exotic black hole horizons.

\subsection{Long-wavelength (GL-type) instability of the symmetric sector}

In this section we study linearized fluctuations of the $\mathbb{Z}_{2}$ symmetry breaking operator $\mathcal{O}_{i}$ in the symmetric phase of the holographic model (2.1). We initialize the symmetric sector of the model as explained in section 3.2 for energy densities above/below the critical one. The bulk scalar $\chi$, dual to an irrelevant operator $\mathcal{O}_{i}$, is initialized as (in $\lambda_{0}=0$ gauge, see appendix A.3)

$$
\left.\chi\right|_{t=0}=\mathcal{A}_{q} \frac{\exp \left(-\frac{1}{r}\right)}{r^{4}} .
$$

To treat symmetry breaking in a linear approximation, we set $\chi(t, x) \equiv 0$ in all dynamical equations, except for the third equation in (3.2) - the only one linear in the field $\chi$ and which determines its dynamics - which is kept unchanged.

Figure 8 presents the linearized fluctuations of the symmetry breaking operator $\mathcal{O}_{i}$ during evolution of the symmetric sector with

$$
\mathcal{E}=0.793642 \mathcal{E}_{\text {crit }} \quad \Longleftrightarrow \quad \mu=-4 \Lambda^{3} .
$$

After a time $t \sim \Lambda^{-1}$ the symmetric sector equilibrates, and $\left\langle\mathcal{O}_{i}\right\rangle$ exhibits an exponential growth with time as it evolves over such state. The growth rate can be extracted at late times [(red) dashed line, right panel]:

$$
\begin{aligned}
\left.\ln \left|\left\langle\mathcal{O}_{i}\right\rangle / \Lambda^{4}\right|\right|_{\text {red line fit }} & =-0.66414(3)+1.0869(7) t \Lambda \\
\operatorname{Im}\left(\omega_{\chi}\right) /\left.\Lambda\right|_{\text {fit }} & =1.0869(7) .
\end{aligned}
$$



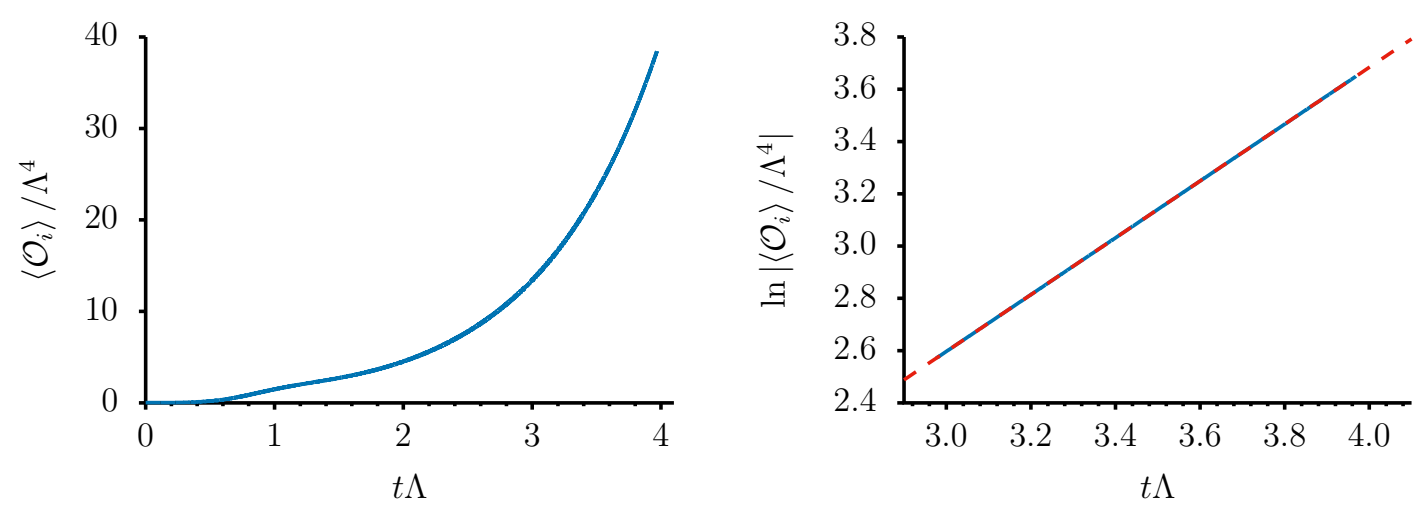

Figure 8. Linearized fluctuations of the symmetry breaking operator $\mathcal{O}_{i}$ during the dynamical evolution of the $\mathbb{Z}_{2}$-symmetric sector of exotic black holes with $\mathcal{E}<\mathcal{E}_{\text {crit }}$. The dashed red line (right panel) is the linearized fit to the exponential growth of $\left\langle\mathcal{O}_{i}\right\rangle$ at late times.
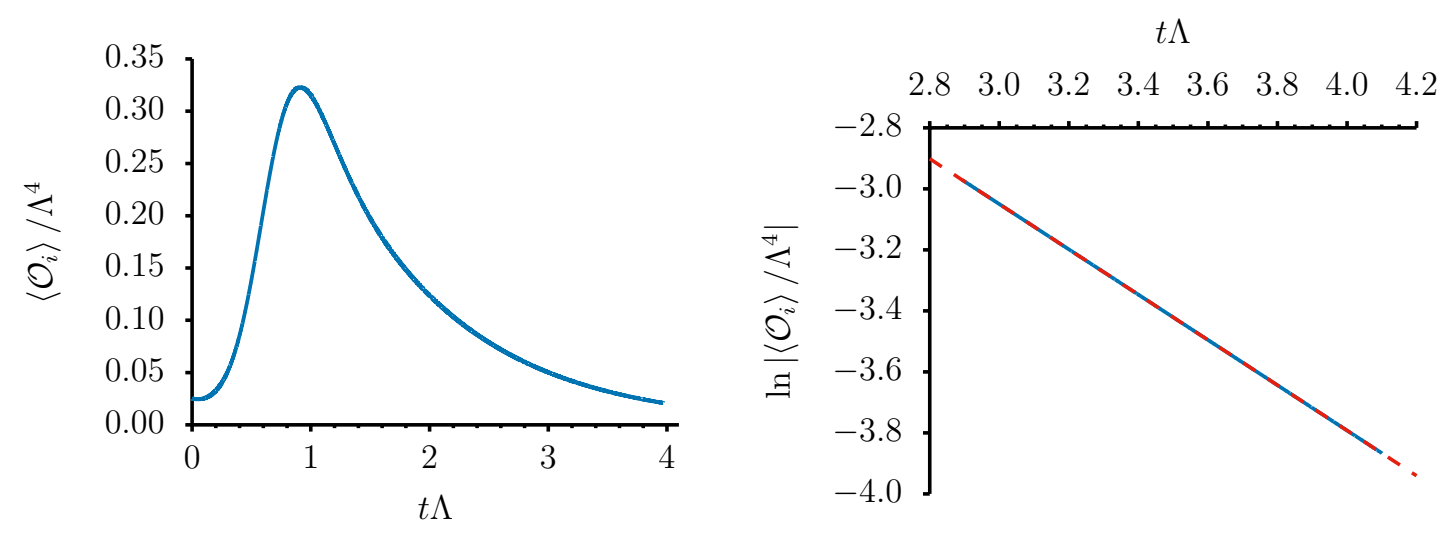

Figure 9. Linearized fluctuations of the symmetry breaking operator $\mathcal{O}_{i}$ during dynamical evolution of the $\mathbb{Z}_{2}$-symmetric sector of exotic black holes with $\mathcal{E}>\mathcal{E}_{\text {crit }}$. The (red) dashed line (right panel) is the linearized fit to the exponential decay of $\left\langle\mathcal{O}_{i}\right\rangle$ at late times.

This is in excellent agreement with the independent computation of the $\chi$-scalar QNM frequencies reported in figure 1 at energy density (3.18):

$$
\frac{\left.\operatorname{Im}\left(\omega_{\chi}\right)\right|_{\mathrm{fit}}}{\left.\operatorname{Im}\left(\omega_{\chi}\right)\right|_{\mathrm{QNM}}}=0.99997(3)
$$

Figure 9 presents the linearized fluctuations of the symmetry breaking operator $\mathcal{O}_{i}$ during evolution of the symmetric sector with

$$
\mathcal{E}=1.1904(6) \mathcal{E}_{\text {crit }} \quad \Longleftrightarrow \quad \mu=-6 \Lambda^{3}
$$

Again, after a time $t \sim \Lambda^{-1}$ the symmetric sector equilibrates, and the evolution of $\left\langle\mathcal{O}_{i}\right\rangle$ over such state exhibits an exponential decay with time. The decay rate can be extracted 
at late times [(red) dashed line, right panel]:

$$
\begin{aligned}
\ln \left|\left\langle\mathcal{O}_{i}\right\rangle / \Lambda^{4}\right| & \\
\operatorname{Im}\left(\omega_{\chi}\right) /\left.\Lambda\right|_{\text {fit }} & =-0.90347(9) .
\end{aligned}
$$

This also agrees with the independent computation of the $\chi$-scalar QNM frequencies reported in figure 1 at energy density (3.21):

$$
\frac{\left.\operatorname{Im}\left(\omega_{\chi}\right)\right|_{\text {fit }}}{\left.\operatorname{Im}\left(\omega_{\chi}\right)\right|_{\mathrm{QNM}}}=1.0000(2) \text {. }
$$

Notice that the fluctuations of $\chi$ do not oscillate (both in the stable, ie. those that give rise to equilibrium, and unstable scenarios), i.e.,

$$
\left.\operatorname{Re}\left(\omega_{\chi}\right)\right|_{\mathrm{QNM}}=0 \text {. }
$$

We believe this is a reflection of the spontaneous character of the symmetry breaking due to these fluctuations at the horizon ${ }^{7}$ together with the boundary conditions adopted.

\subsection{Fully non-linear evolutions of stable and unstable black holes}

We now turn our attention to the fully non-linear behavior. Thanks to the simulations' ability to account for the backreaction of the field $\chi$ a rich phenomenology is uncovered. To aid in the interpretation of the results, we monitor several quantities:

- The dynamical behavior of $p_{2}$ and $q_{4}$.

- The area of the Apparent and Event horizons (see appendix A.5).

- The behavior of the Kretschmann curvature scalar $K=R_{a b c d} R^{a b c d}$ (normalized by the value of $K$ for pure AdS).

As a first case of study, we confirm that for $\mathcal{E}>\mathcal{E}_{\text {crit }}$ the behavior observed is consistent with that captured by the linearized analysis described in section 3.3. For this case, the system asymptotically approaches a stationary hairy black hole which is evidenced by a non-zero asymptotic value of $p_{2}$ as illustrated in figure 10 as well as the behavior of the normalized curvature scalar $K$ shown in figure 11. This figure, also shows that at late times the event and apparent horizon coincide and remain stationary.

On the other hand, the case where $\mathcal{E}<\mathcal{E}_{\text {crit }}$ - identified in the previous section as unstable - leads to a markedly different behavior. For concreteness, we concentrate on the particular case defined by the following configuration.

- Energy density (with $\Lambda=1$ )

\footnotetext{
${ }^{7}$ Similar phenomena was observed in [24].
}

$$
\mathcal{E}=0.793642 \mathcal{E}_{\text {crit }} \quad \Longleftrightarrow \quad \mu=-4 \Lambda^{3} .
$$



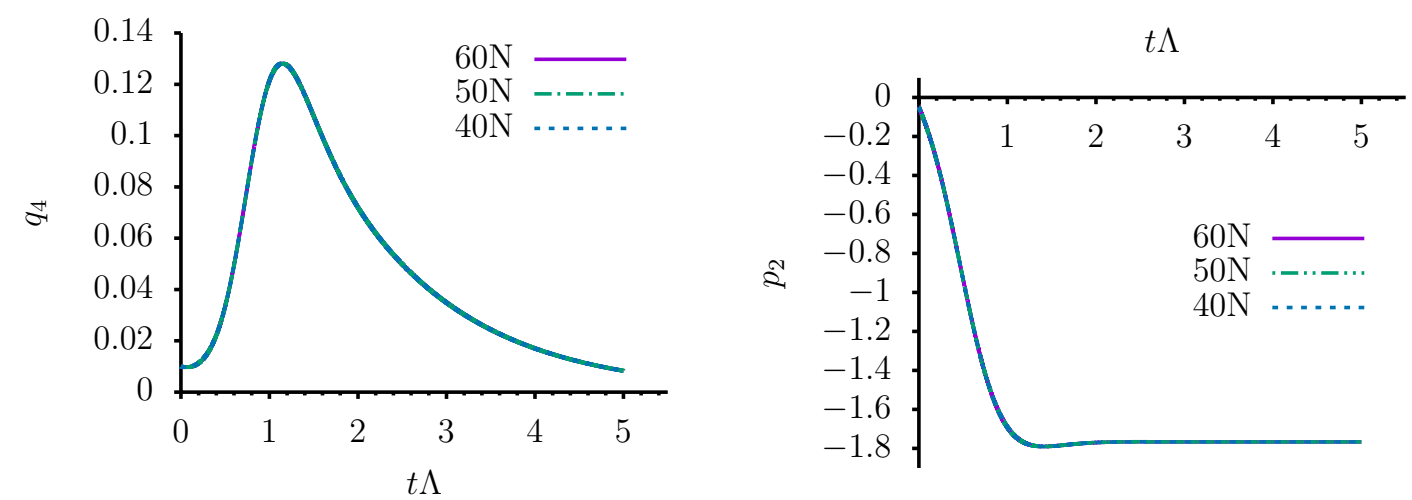

Figure 10. $p_{2}$ and $q_{4}$ vs. time for the stable case.
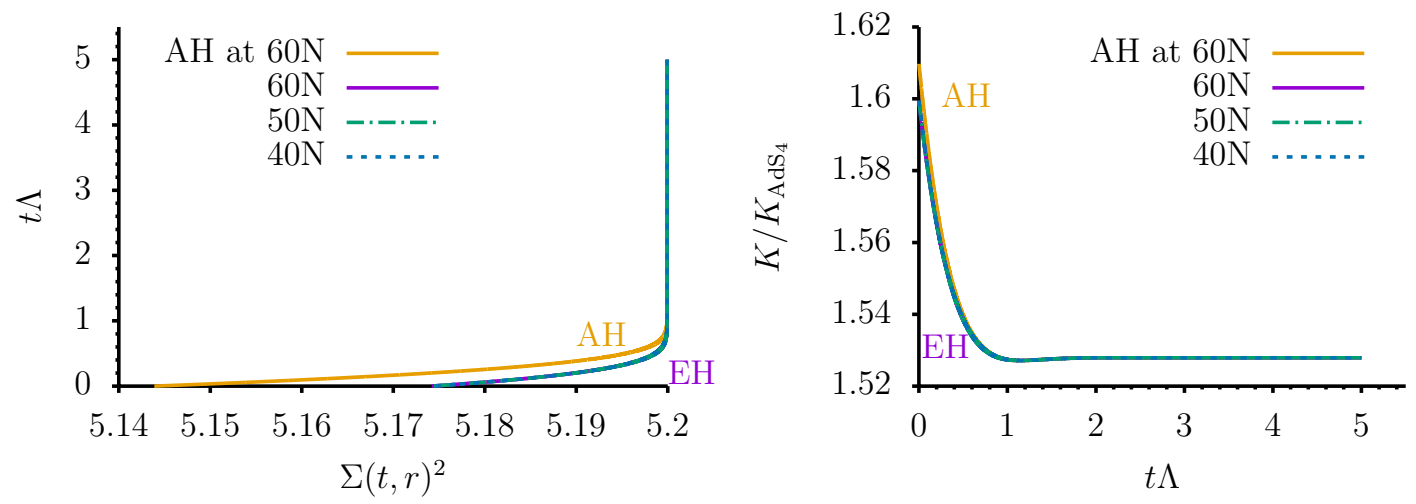

Figure 11. Area and Kretschmann for stable case.

- Initial conditions are chosen describing a perturbed black hole with both non-zero $\phi$ and $\chi$ (as detailed in appendix A.3) with

$$
\mathcal{A}_{p}=1.0, \quad \mathcal{A}_{q}=0.01
$$

Under these conditions, the system gives rise to a rich — and very rapidly evolving dynamics - which we have confirmed through extensive convergent studies. For instance, by inspection of results obtained with different number of collocation points $(N=20,30,40,50,60$ points), use of adaptive time-stepping to capture the increasing faster dynamics observed, and employing a different coordinate condition (setting $\lambda(t)=0$ ) which does not keep the apparent horizon at a fixed location as done in [25]. All these studies confirm the observed behavior that we describe next.

As the field $\phi$ "rolls down" the unbounded potential, the energy gained impacts the dynamics of its normalizable coefficient $\left(p_{2}\right)$ as well as the normalizable coefficient of $\chi$ which grows without bounds as shown in figure 12. This behavior is evidenced in the black hole, which grows fast and eventually reaches the AdS boundary in finite asymptotic time as illustrated in figure 13. The figure shows both the apparent horizon (AH), and the event horizon (EH) as well as the curvature scalar evaluated on them. Clearly, as time progresses, the $\mathrm{AH}$ approaches the $\mathrm{EH}$ and both asymptote to infinite size in a finite amount of time. 

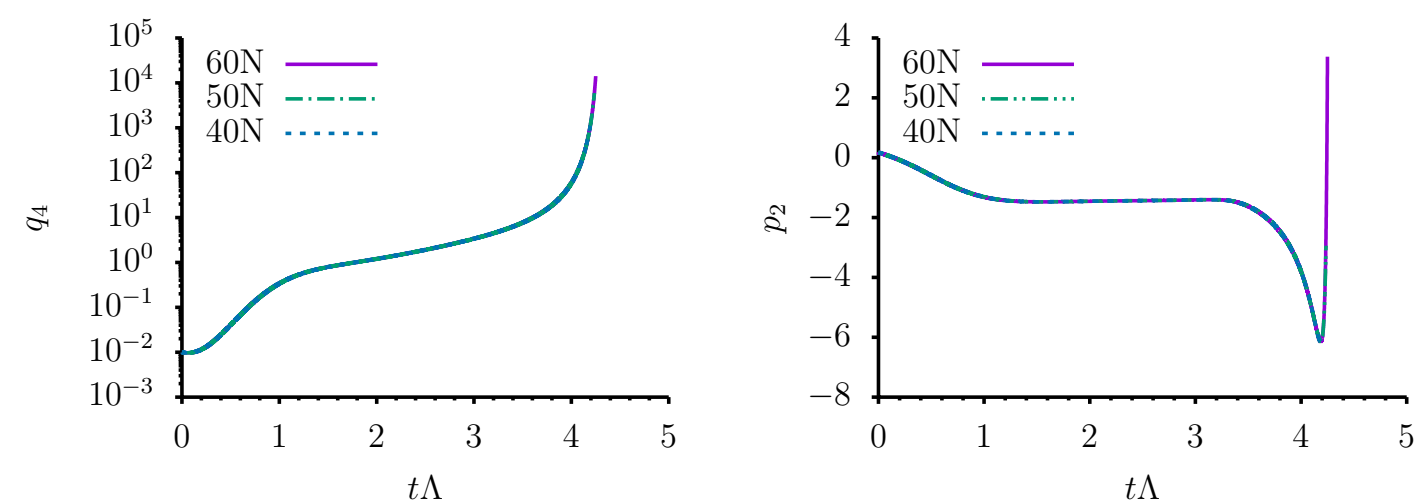

Figure 12. $p_{2}$ and $q_{4}$ vs. time for the unstable case.

This asymptotic behavior can be fit by the expression

$$
\Sigma_{\mathrm{EH}}^{2} \propto 1 /\left(a+b t \Lambda+c(t \Lambda)^{2}\right),
$$

with the following coefficients $\{a=3.934(5), b=-1.811(5)$ and $c=0.2084(5)\}$. This fit indicates a finite time divergence at $t \approx 4.30$. We find a similar asymptotic behaviour for the Kretschmann scalar evaluated at the horizon with $K \simeq \Sigma_{\mathrm{EH}}^{3}$, as seen in figure 14 . Thus, at late times,

$$
K_{\mathrm{EH}} \propto \Sigma_{\mathrm{EH}}^{3} \propto\left(\frac{1}{a+b t \Lambda+c(t \Lambda)^{2}}\right)^{\frac{3}{2}} ;
$$

consequently, $K_{\mathrm{EH}}$ diverges in finite time at the boundary of AdS. Additionally, the (normalized) scalar curvatures on the $\mathrm{AH}$ and $\mathrm{EH}$ diverge with $K_{\mathrm{EH}} \leq K_{\mathrm{AH}}$. Naturally, the code is eventually unable to keep up with the radically rapid dynamics which requires ever smaller timesteps to capture the following behavior. Nevertheless, we have been able to extract convergent solutions up to a sufficiently late stage to understand the behavior and fate of the spacetime. The picture that arises is that the spacetime explores arbitrarily large curvatures in finite time, and outgoing null geodesics emanating from such regions reach the boundary of AdS in finite asymptotic time as indicated in figure 15. This behavior would violate the spirit of the weak cosmic censorship conjecture, in that far observers can be reached by signals emanating from arbitrarily curved spacetime regions, and is similar to that recently reported in [26].

\section{Conclusions}

In this work we studied an interesting instability of the black hole horizons, observed first in [8]: below some critical energy density the horizon is unstable with respect to fluctuations spontaneously breaking a discrete symmetry. However, there is no static endpoint associated with the nonlinear build-up of the symmetry breaking condensate (scalar hair at the horizon), as opposed to typical constructions of holographic superconductors [3]. The instability is perturbative in nature (i.e., describing a second order phase transition), and is triggered by an arbitrary small amplitude of the symmetry breaking mode, provided the conserved energy density of the state $\mathcal{E}$ is below a critical energy density. As a result, 

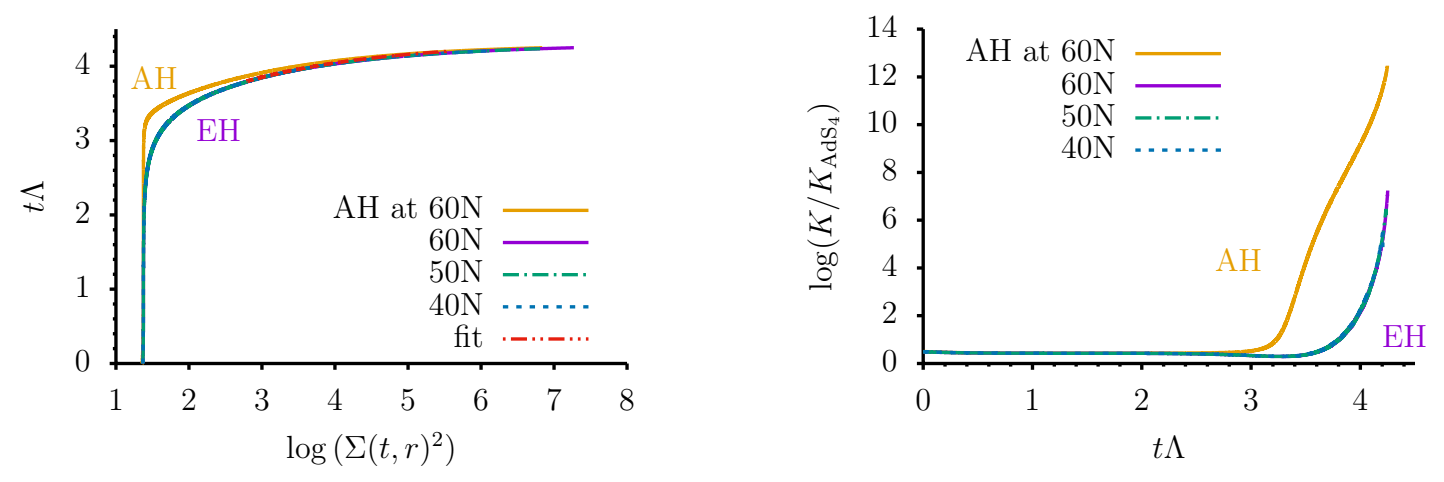

Figure 13. Area and Kretschmann for unstable case.

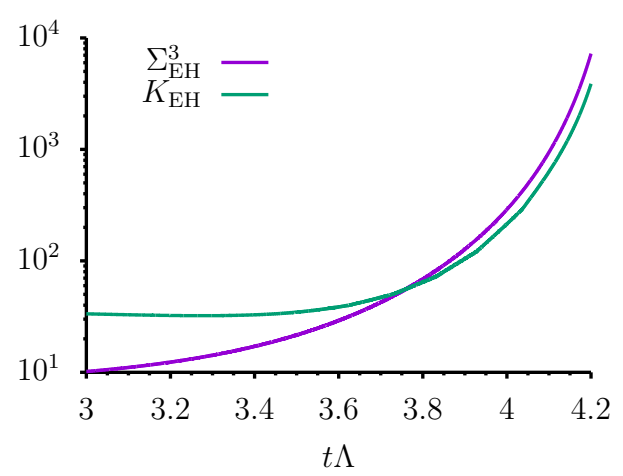

Figure 14. Similar asymptotic behaviour of the Kretschmann scalar and $\Sigma^{3}$ at the event horizon.

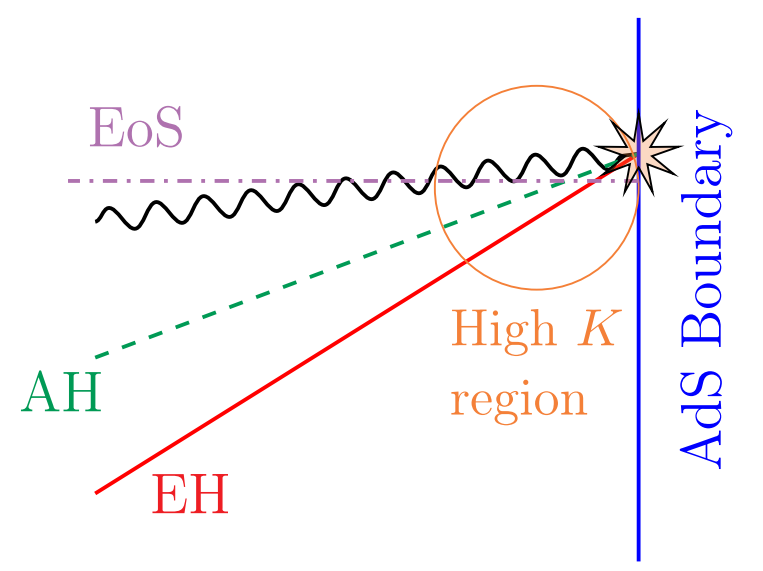

Figure 15. Schematic spacetime diagram. As time progresses, the apparent horizon approaches the event horizon in finite time. The scalar curvature diverges and arbitrarily high curvature regions can be identified by asymptotic observers at finite times. In the diagram, "EoS" refers to the "End of the Simulation" while the star refers to the blow up of the Kretschmann at the boundary in finite time.

the onset of the instability, and dynamics close to it, can not be affected by higher-order nonlinearities in the gravitational scalar potential as long as the amplitude of unstable modes remain small. Additionally, the instability initiates in the long wavelength regime, 
i.e., at small bulk curvature, and thus can not be removed by higher derivative corrections to the gravitational effective action.

We studied the future development of the instability, using a characteristic formulation of asymptotically anti-de Sitter gravitational dynamics [14], and argued that, at the classical level, the end point of the instability induces a curvature singularity at finite asymptotic time. Specifically, we demonstrated an apparently unbounded growth of the Kretschmann scalar in the bulk (e.g., evaluated at the location of the apparent horizon). Thus, our model (2.1) provides a simple example of arbitrarily large curvatures arising in asymptotically anti-de Sitter space times at sufficiently late times.

While the analysis of this work is focused on a specific phenomenological model of gauge theory/gravity correspondence, represented by the effective gravitational action (2.1), the phenomena described, i.e., horizon instability without the static end point, is realized within bona fide holographic correspondence scenarios (e.g., [9, 13]). We expect that curvature singularities also arise in those models as well. ${ }^{8}$ The observation that curvature singularities might arise dynamically implies that consistent truncations of string theory and supergravity, while suitable to address static states in the theory, may fail in dynamical settings - when the evolution enters the regime of highly curved geometry; and in such cases stringy corrections will be important.

What are the holographic implications of a singularity developing evolution for the boundary gauge theory? A standard lore is that states of a closed non-integrable interacting system with large number of degrees of freedom should dynamically equilibrate [28]. In the context of holography, early indications supported this for generic far-from-equilibrium, arbitrary low-energy states of strongly coupled conformal gauge theories, even when the dynamical evolution of these states was artificially restricted to symmetric submanifolds of the full phase space of the theory ${ }^{9}[29,30]$. Shortly thereafter it was argued [31-33] that in fact symmetric phase space of holographic conformal field theories has islands of stability that never equilibrate. In this study we identified yet another possibility: initial states of holographic strongly coupled gauge theories, well described classically in the gravitational frame, evolve to a singularity in finite time. Singularity is a signature for a breakdown of an approximation, and we see two possible reasons. First, it is possible the singularity is an artifact of our restriction of the state evolution to symmetric submanifolds of the full phase space of the theory and that symmetry breaking modes would allow for a smooth evolution. As we discuss below, hydrodynamic modes in the system can be gapped, leading to the same qualitative behaviour. It is more difficult to argue for the absence of light modes spontaneously breaking internal symmetries - the singularity observed might be an indication that some of these symmetries must be dynamically broken during the evolution (similar ideas were proposed in $[27,34]$ ). Second, the state evolution in the gravitational frame of the holography might not be always semiclassical.

We find it important to discuss another possible limitation of the study carried out here and its conclusions. We restricted the dynamics in our model to preserve boundary

\footnotetext{
${ }^{8}$ It is a straightforward exercise to examine this in a holographic model [9]; dynamics of small black hole localization in $A d S_{5} \times S^{5}$ is much mode difficult [27].

${ }^{9}$ The states in question were spatially isotropic, and invariant under all global symmetries, i.e., the R-symmetry.
} 
homogeneity and isotropy. One might argue that the physical phenomena discussed here arise as a consequence of such a restriction, and that a sufficiently generic initial state would smoothly evolve to an end point where these symmetries are spontaneously broken. We do not have a full answer to this question - gravitational simulations in the presence of spatial inhomogeneities are beyond the scope of this paper. At the very least, in the holographic model studies in [35] there is an exotic instability discussed, without the spatially modulated endpoint as well. ${ }^{10}$ Since the instability and the evolution towards the singularity in our model can be triggered by arbitrarily small amplitude fluctuations of the $\chi$-mode, i.e., energetically arbitrary close to the critical point, the potentially physicsmodifying hydrodynamics modes can be gapped, rendering them irrelevant to the question as to whether or not the singularity observed is physical. To demonstrate this, we modified our model with a boundary with topology $R^{2} \rightarrow S^{2}$. All the main features described in the former case remain in the latter, in particular: there is a horizon instability, there is no static end point below some critical energy density associated with the onset of the perturbative instability, the low-energy $\mathrm{SO}(3)$-invariant states evolve to a singular solution. It would be interesting to explore in details the role of additional massless fields at the threshold of instability, and their effect on the singularity development.

A feature of the bulk scalar potential of our holographic model (2.1) is that it is unbounded in the $\chi$-direction (recall that the nonlinear coupling $g<0$ ). We study in appendix B a modification of the model which "bounds" the $\chi$-potential with higher order, nonlinear in $\chi$, interactions. ${ }^{11}$ Of course, the linearized instability is unaffected; likewise, the unstable phase with $\left\langle\mathcal{O}_{i}\right\rangle \neq 0$ for $\mathcal{E}>\mathcal{E}_{\text {crit }}$ is unchanged qualitatively (close to $\mathcal{E}_{\text {crit }}$ the higher-order nonlinear terms in the gravitational potential are suppressed). However, we find a new static black hole phase with $\left\langle\mathcal{O}_{i}\right\rangle \neq 0$, that exists both for $\mathcal{E}<\mathcal{E}_{\text {crit }}$ and $\mathcal{E}>\mathcal{E}_{\text {crit }}$ for the bounded potentials and, at least in the vicinity of perturbative instability, has higher entropy density than the symmetric phase. Unlike the exotic branch of the black holes, this new phase does not bifurcate from the onset of long-wavelength instability of the symmetric phase. For $\mathcal{E}<\mathcal{E}_{\text {crit }}$ this new static phase is always the end point of the evolution; for $\mathcal{E}>\mathcal{E}_{\text {crit }}$ the new symmetry broken phase can only be reached if the initial amplitude of the symmetry breaking fluctuations is sufficiently large - the symmetry broken phase is a potential barrier separated from the symmetric phase whenever $\mathcal{E}>\mathcal{E}_{\text {crit }}$. Our model (2.1) is a phenomenological example of the holographic correspondence, thus one might worry whether curvature diverging scenarios described here is realized in genuine (top-down) holographic dualities. We believe the answer to the question is in the affirmative:

- First, the unbounded potentials are ubiquitous in holography - a typical example is a well-studied $\mathcal{N}=2^{*}$ holography [36-38], where the bulk gravitational scalars $\{\alpha, \chi\}$ effective action takes form,

$$
\begin{aligned}
S_{\mathcal{N}=2^{*}}^{\text {scalar }} & \sim \frac{1}{16 \pi G_{5}} \int \mathrm{d}^{5} x \sqrt{-g}\left(-12(\partial \alpha)^{2}-4(\partial \chi)^{2}-V\right), \\
V(\alpha, \chi) & =-e^{-4 \alpha}-2 e^{2 \alpha} \cosh 2 \chi+\frac{1}{4} e^{8 \alpha} \sinh ^{2} 2 \chi .
\end{aligned}
$$

\footnotetext{
${ }^{10}$ We would like to thank Ben Withers for bringing the reference to our attention.

${ }^{11}$ We would like to thank Jorge Santos for raising the issue of the unboundedness of the scalar potential in our model with its potential effect on the singular evolution that prompted this analysis.
} 
The reason why the scalar potentials in supergravity constructions can be unbounded comes from the fact that they arise from the superpotential as (for the $\mathcal{N}=2^{*}$ example (4.1))

$$
\begin{aligned}
V & =\frac{1}{16}\left[\frac{1}{3}\left(\frac{\partial W}{\partial \alpha}\right)^{2}+\left(\frac{\partial W}{\partial \chi}\right)^{2}\right]-\frac{1}{3} W^{2}, \\
W & =-e^{-2 \alpha}-\frac{1}{2} e^{4 \alpha} \cosh (2 \chi) .
\end{aligned}
$$

It is the $-\frac{1}{3} W^{2}$ contribution to $V$ in (4.2) that is responsible for the unboundedness of $V$.

- Second, the scalar potential in the top-down embedding of the exotic black hole phenomena [8] constructed in [9] (see eq. (2.32) there) is unbounded from below:

$$
V(\varphi)=-2(2+\cosh (2 \varphi))
$$

There are lots of open questions left for the future. It would be interesting to understand whether the divergent curvature scenario proposed here is universal. Is it possible to understand analytically the approach towards the singularity as in explorations of the BKL conjecture? (e.g. [39]). The link between the boundedness of the gravitational potential and the singular evolution should be studied in more detail. It is certainly important to understand the consequences of the diverging curvature for the boundary gauge theory. Is there a QFT-solvable holographic example that captures the proposed singular behavior?

\section{Acknowledgments}

Research at Perimeter Institute is supported by the Government of Canada through Industry Canada and by the Province of Ontario through the Ministry of Research \& Innovation. This work was further supported by NSERC through the Discovery Grants program (AB and LL), by CIFAR (LL) and by CONACyT-Mexico (PB).

\section{A Numerical setup}

We adapt the characteristic formulation of [14] for the numerical solution of (3.2)-(3.4).

\section{A.1 Field redefinitions and the code equations}

We introduce a new radial coordinate

$$
x \equiv \frac{1}{r} \in[0,1], \quad d_{+}=\partial_{t}+A(t, r) \partial_{r} \rightarrow \partial_{t}-x^{2} A(t, x) \partial_{x},
$$

maintaining ${ }^{\prime} \equiv \partial_{x}$ and ${ }^{*} \equiv \partial_{t}$, and redefine the fields

$$
\left\{\phi, \chi, \Sigma, A, d_{+} \phi, d_{+} \chi, d_{+} \Sigma\right\} \rightarrow\{p, q, \sigma, a, d p, d q, d \sigma\}
$$


as follows

$$
\begin{aligned}
\phi(t, x) & =x p_{1}+x p(t, x), \\
\chi(t, x) & =x^{3} q(t, x), \\
\Sigma(t, x) & =\frac{1}{x}+\sigma(t, x), \\
A(t, x) & =a(t, x)+\frac{1}{2} \Sigma(t, x)^{2}, \\
d_{+} \phi(t, x) & =-\frac{p_{1}}{2}+x d p(t, x), \\
d_{+} \chi(t, x) & =x^{3} d q(t, x), \\
d_{+} \Sigma(t, x) & =x d \sigma(t, x)+\frac{1}{2} \Sigma(t, x)^{2}-\frac{p_{1}}{12} d_{+} \phi(t, x)+\frac{p_{1}^{2}}{48} .
\end{aligned}
$$

Using (3.5), we find the asymptotic boundary expansion $x \rightarrow 0_{+}$for the new fields:

$$
\begin{aligned}
p & =p_{2}(t) x+\mathcal{O}\left(x^{2}\right), & q & =q_{4}(t) x+\mathcal{O}\left(x^{2}\right), \\
d p & =-p_{2}(t)-p_{1} \lambda(t)+\mathcal{O}(x), & d q & =-2 q_{4}(t)+\mathcal{O}(x), \\
\sigma & =\lambda(t)-\frac{p_{1}^{2}}{8} x+\mathcal{O}\left(x^{2}\right), & d \sigma & =\mu+\mathcal{O}(x), \\
a & =-\dot{\lambda}(t)+\left(\mu-\frac{p_{1}^{2}}{12} \lambda(t)-\frac{p_{1}}{12} p_{2}(t)\right) & x+\mathcal{O}\left(x^{2}\right) . &
\end{aligned}
$$

In new variables (A.3), the equations of motion used to evolve the system take form:

$$
\begin{aligned}
& {\left[\partial_{x x}^{2}+\frac{2}{x} \partial_{x}+\frac{x^{4}}{4}\left(3 q+x q^{\prime}\right)^{2}+\frac{1}{4}\left(p_{1}+p+x p^{\prime}\right)^{2}\right] \sigma=J_{\sigma}, } \\
& J_{\sigma}\left\{p, p^{\prime}, q, q^{\prime}\right\}=-\frac{x^{3}}{4}\left(3 q+x q^{\prime}\right)^{2}-\frac{p^{\prime}}{4}\left(x p^{\prime}+2 p+2 p_{1}\right)-\frac{1}{4 x}\left(p+p_{1}\right)^{2}, \\
& {\left[\partial_{x}+\frac{12 \sigma+12 x \sigma^{\prime}-}{12\left(1+x p_{1}\left(p_{1}+p+x p^{\prime}\right)\right.}\right] d p+\left[\frac{x\left(p_{1}+p+x p^{\prime}\right)}{1+x \sigma}\right] d \sigma=J_{2}, } \\
& {\left[\partial_{x}+\frac{12 \sigma+12 x \sigma^{\prime}+}{12\left(1+x p_{1}\left(p_{1}+p+x p^{\prime}\right)\right.}\right] d \sigma+\left[-\frac{x p_{1}^{2}\left(p_{1}+p+x p^{\prime}\right)}{144(1+x \sigma)}\right] d p=J_{3}, } \\
& J_{2}\left\{p, p^{\prime}, q, \sigma, \sigma^{\prime}\right\}= \frac{1}{1+x \sigma}\left(-\frac{1}{16} p^{\prime}\left(p_{1}^{2}+8 \sigma^{2}\right) x-\frac{1}{16}\left(p_{1}^{2}+8 \sigma^{2}\right)\left(p+p_{1}\right)+\frac{1}{2} p_{1} \sigma^{\prime}\right. \\
&\left.-p^{\prime} \sigma-\frac{p^{\prime}}{2 x}+\frac{p}{2 x^{2}}\right)-\left(p+p_{1}\right) q^{2} g x^{4}, \\
& J_{3}\left\{p, p^{\prime}, q, \sigma, \sigma^{\prime}\right\}= \frac{1}{1+x \sigma}\left(-\frac{p^{\prime} p_{1}}{192}\left(p_{1}^{2}+8 \sigma^{2}\right) x-\frac{p+p_{1}}{192}\left(48 p \sigma^{2}+p_{1}^{3}+56 p_{1} \sigma^{2}\right)\right. \\
&-\frac{\sigma^{\prime}}{48}\left(p_{1}^{2}+72 \sigma^{2}\right)-\frac{p_{1} p^{\prime} \sigma}{12}+\frac{1}{x}\left(-\frac{\sigma}{2}\left(p+p_{1}\right)^{2}-3 \sigma \sigma^{\prime}-\frac{p^{\prime} p_{1}}{24}\right) \\
&\left.+\frac{1}{x^{2}}\left(-\frac{p^{2}}{4}-\frac{11}{24} p p_{1}-\frac{3}{16} p_{1}^{2}-\frac{3}{2} \sigma^{\prime}\right)\right)+\frac{g}{4} \sigma\left(p+p_{1}\right)^{2} q^{2} x^{5} \\
&+\frac{g}{12}\left(p+p_{1}\right)\left(3 p+2 p_{1}\right) q^{2} x^{4}+\frac{1}{2} \sigma q^{2} x^{3}+\frac{1}{2} q^{2} x^{2},
\end{aligned}
$$




$$
\begin{aligned}
& {\left[\partial_{x}+\frac{2+3 x \sigma+x^{2} \sigma^{\prime}}{x(1+x \sigma)}\right] d q+\left[-\frac{x^{2} p_{1} q^{\prime}+3 x p_{1} q}{12(1+x \sigma)}\right] d p+\left[\frac{x^{2} q^{\prime}+3 x q}{1+x \sigma}\right] d \sigma=J_{4},} \\
& J_{4}\left\{p, q, q^{\prime}, \sigma\right\}=\frac{1}{1+x \sigma}\left(-\left(q \sigma g\left(p+p_{1}\right)^{2}+\frac{1}{2} \sigma^{2} q^{\prime}+\frac{1}{16} p_{1}^{2} q^{\prime}\right) x\right. \\
& -\frac{q}{16}\left(16 g\left(p+p_{1}\right)^{2}+3 p_{1}^{2}+24 \sigma^{2}\right)-\sigma q^{\prime} \\
& \left.+\frac{1}{x}\left(-5 \sigma q-\frac{1}{2} q^{\prime}\right)-\frac{7 q}{2 x^{2}}\right), \\
& {\left[\partial_{x x}^{2}+\frac{2}{x} \partial_{x}\right] a+\left[\frac { 1 } { ( 1 + x s ) ^ { 2 } } \left(-\frac{1}{2} p^{\prime} \sigma^{2} x^{2}+\left(-\frac{1}{2} \sigma^{2}\left(p+p_{1}\right)-p^{\prime} \sigma-\frac{1}{6} p_{1} \sigma^{\prime}\right) x\right.\right.} \\
& \left.\left.-\sigma\left(p+p_{1}\right)-\frac{p^{\prime}}{2}-\frac{3 p+2 p_{1}}{6 x}\right)\right] d p+\left[\frac{2\left(x^{2} \sigma^{\prime}-1\right)}{x(1+x s)^{2}}\right] d \sigma+\left[-\frac{x^{3}\left(x q^{\prime}+3 q\right)}{2}\right] d q=J_{5}, \\
& J_{5}\left\{p, p^{\prime}, q, q^{\prime}, \sigma, \sigma^{\prime}\right\}=\frac{1}{(1+x s)^{2}}\left(\frac{\sigma^{4}}{4}\left(p^{\prime}\right)^{2} x^{4}+\frac{\sigma^{3}}{2} p^{\prime}\left(p \sigma+p_{1} \sigma+2 p^{\prime}\right) x^{3}\right. \\
& +\left(\frac{1}{4} \sigma^{4}\left(p+p_{1}\right)^{2}+2 p^{\prime} \sigma^{3}\left(p+p_{1}\right)+\frac{3}{2}\left(p^{\prime}\right)^{2} \sigma^{2}-\left(\sigma^{\prime}\right)^{2} \sigma^{2}\right) x^{2} \\
& +\left(\sigma^{3}\left(p+p_{1}\right)^{2}+\frac{1}{4} p^{\prime} \sigma^{2}\left(12 p+11 p_{1}\right)+\left(p^{\prime}\right)^{2} \sigma-2\left(\sigma^{\prime}\right)^{2} \sigma\right) x \\
& +\frac{1}{4} \sigma^{2}\left(p+p_{1}\right)\left(6 p+5 p_{1}\right)+\frac{\sigma}{2} p^{\prime}\left(4 p+3 p_{1}\right)+\frac{1}{8} \sigma^{\prime}\left(-p_{1}^{2}+8 \sigma^{2}\right) \\
& +\frac{1}{4}\left(p^{\prime}\right)^{2}-\left(\sigma^{\prime}\right)^{2}+\frac{1}{x}\left(\frac{\sigma}{2}\left(p+p_{1}\right)\left(2 p+p_{1}\right)+\frac{1}{4} p^{\prime}\left(2 p+p_{1}\right)+2 \sigma \sigma^{\prime}\right) \\
& \left.+\frac{1}{x^{2}}\left(\frac{p^{2}}{4}+\frac{p p_{1}}{4}+\frac{p_{1}^{2}}{8}+\sigma^{\prime}\right)\right)+\frac{1}{4} x^{2}\left(q^{\prime} x+3 q\right)^{2}(\sigma x+1)^{2}, \\
& \dot{p}=d p+\frac{1}{2} p^{\prime}\left(\sigma^{2}+2 a\right) x^{2}+\left(\frac{1}{2}\left(p+p_{1}\right)\left(\sigma^{2}+2 a\right)+p^{\prime} \sigma\right) x+\left(p+p_{1}\right) \sigma+\frac{p^{\prime}}{2}+\frac{p}{2 x}, \\
& \dot{q}=d q+\frac{1}{2} q^{\prime}\left(\sigma^{2}+2 a\right) x^{2}+\left(\frac{3}{2}\left(\sigma^{2}+2 a\right) q+\sigma q^{\prime}\right) x+3 \sigma q+\frac{q^{\prime}}{2}+\frac{3 q}{2 x} .
\end{aligned}
$$

Numerical code is organized as follows.

- [Step 1]: assume that at a time step $t$ we have profiles

$$
\left\{p(t, x), q(t, x), p^{\prime}(t, x), q^{\prime}(t, x)\right\} \quad \text { and } \quad \lambda(t) .
$$

- [Step 2]: we solve linear in $\sigma$ equation (A.5), subject to boundary conditions

$$
\sigma(t, x=0)=\lambda(t), \quad \sigma^{\prime}(t, x=0)=-\frac{p_{1}^{2}}{8} .
$$

- [Step 3]: we solve linear in $\{d p, d \sigma\}$ system (A.6), subject to the boundary conditions

$$
d p(t, x=0)=-p^{\prime}(t, x=0)-\lambda(t) p_{1}, \quad d \sigma(t, x=0)=\mu .
$$

- [Step 4]: we solve linear in $d q$ equation (A.7), subject to the boundary conditions

$$
d q(t, x=0)=-2 q^{\prime}(t, x=0) .
$$


- [Step 5]: we solve linear in $a$ equation (A.8), subject to the boundary conditions

$$
a^{\prime}(t, x=0)=\mu-\frac{p_{1}^{2}}{12} \lambda(t)-\frac{p_{1}}{12} p^{\prime}(t, x=0), \quad a(t, x=1)=a^{h} .
$$

The value $a^{h}$ is determined from the stationarity of the apparent horizon at $x=1$ as explained in the following subsection.

- [Step 6]: we use evolution equations (A.9), along with (see (A.4))

$$
\dot{\lambda}(t)=-a(t, x=0),
$$

to compute

$$
\{p(t+d t, x), q(t+d t, x), \lambda(t+d t) .
$$

After computing the radial coordinate derivatives $\left\{p^{\prime}(t+d t, x), q^{\prime}(t+d t, x)\right\}$, we repeat [Step 1].

Notice that the first equation in (3.2) is redundant in our numerical procedure: rather than propagating in time $\Sigma$, we compute it from the constraint (3.3) at each time step; nonetheless, we monitor the consistency of that equation during the evolution.

Implementing the code ${ }^{12}$ we use spectral methods for the radial coordinate integration, [Step 2]-[Step 5]. Singularities of the equations at the boundary collocation point $x=0$ are resolved using the corresponding boundary conditions instead. We use fourth-order Runge-Kutta method for the time evolution, [Step 6].

\section{A.2 Apparent horizon and the boundary condition for $a$}

Our numerical implementation requires an independent computation of $a^{h} \equiv a(t, x=1)$ (see (A.14)), given radial profiles $\left\{p, p^{\prime}, q, q^{\prime}, s, s^{\prime}, d p, d s, d q\right\}$ and the diffeomorphisms parameter $\lambda$ at time $t$. Following [14], this is done by enforcing the time-independent location of the horizon. Apparent horizon is located as $x=x_{h}$ such that

$$
\left.d_{+} \Sigma(t, x)\right|_{x=x_{h}}=0 \text {. }
$$

Assuming $x_{h}=1, \frac{d x_{h}}{d t}=0$, and using equations of motion (A.5)-(A.9) we compute $a^{h}$ from

$$
\left.\partial_{t} d_{+} \Sigma\left(t, x_{h}\right)\right|_{x_{h}=1}=0 .
$$

Denoting

$$
\left.\left\{p^{h}, d p^{h}, q^{h}, d q^{h}, \sigma^{h}\right\} \equiv\{p, d p, q, d q, \sigma\}\right|_{(t, x=1)}
$$

we find

$$
\begin{aligned}
a^{h}= & \frac{1}{4}\left(\left(g\left(q^{h}\right)^{2}-1\right)\left(p_{1}+p^{h}\right)^{2}+2\left(q^{h}\right)^{2}-6\right)^{-1}\left(\left(2 d p^{h}-p_{1}\right)^{2}+4\left(d q^{h}\right)^{2}\right. \\
& \left.+2\left(\left(p^{h}+p_{1}\right)^{2}-2\left(q^{h}\right)^{2}+6\right)\left(\sigma^{h}+1\right)^{2}-2\left(q^{h}\right)^{2}\left(\sigma^{h}+1\right)^{2}\left(p_{1}+p^{h}\right)^{2} g\right) .
\end{aligned}
$$

\footnotetext{
${ }^{12}$ Code implementation is similar to the one used in [40].
} 


\section{A.3 Initial conditions}

To evolve (A.5)-(A.9) one has to provide data, at $t=0$ as required by [Step 1], see (A.10). In particular, we need to specify $\lambda_{0} \equiv \lambda(t=0)$. Once again, we follow [14].

Recall that both $\phi$ and $\chi$ are left invariant under the reparametrization transformations:

$$
\frac{1}{x} \rightarrow \frac{1}{x}+\lambda_{0}
$$

To maintain this invariance we specify initial conditions for $\{p, q\}$ (in $\lambda_{0}$-invariant way) in terms of two amplitudes $\left\{\mathcal{A}_{p}, \mathcal{A}_{q}\right\}$ :

$$
\begin{aligned}
\left.p\right|_{t=0} & =\mathcal{A}_{p} \frac{x}{\left(1+x \lambda_{0}\right)^{2}} \exp \left[-\frac{x}{1+x \lambda_{0}}\right]-\frac{p_{1} \lambda_{0} x}{1+x \lambda_{0}}, \\
\left.q\right|_{t=0} & =\mathcal{A}_{q} \frac{x}{\left(1+x \lambda_{0}\right)^{4}} \exp \left[-\frac{x}{1+x \lambda_{0}}\right] .
\end{aligned}
$$

We then proceed as follows: ${ }^{13}$

- given $\left\{\mathcal{A}_{p}, \mathcal{A}_{q}\right\}$ we set $\lambda_{0}=0$ and perform [Step 2] (A.11) and [Step 3] (A.12);

- having enough data, we follow (A.3) to compute the profile $d_{+} \Sigma(t=0, x)$;

- we find numerically the root $x=x_{0}$ of the equation

$$
\left.d_{+} \Sigma(t=0, x)\right|_{x=x_{0}}=0
$$

- we set the trial value of $\lambda_{0}$ as

$$
\lambda_{0}=\frac{1}{x_{0}}-1,
$$

which (apart from the numerical errors) would guarantee that the corresponding location of the apparent horizon is now at $x=1$;

- the trial value (A.24) is further adjusted repeatedly performing [Step 2] and [Step 3] to achieve

$$
\left.d_{+} \Sigma(t=0, x)\right|_{x=1}=0
$$

at a high accuracy.

\section{A.4 Convergence tests}

We performed self-convergence tests to verify the validity of the obtained numerical solutions. In particular, we study each configuration numerically under different number of collocation points $N=20,30,40,50,60,80$. We monitored the convergence of the residuals of the constraint equations to zero as well as each evolved field (and computing selfconvergence test by a suitable interpolation onto a finite difference grid). Additionally, we confirmed convergence of the location of the event horizon and the Kretschmann scalar at both the apparent and event horizons. As an illustration, figure 16 displays $K_{\mathrm{AH}}$ for

\footnotetext{
${ }^{13}$ For this procedure the integration range over the radial coordinate $x$ might exceed unity.
} 

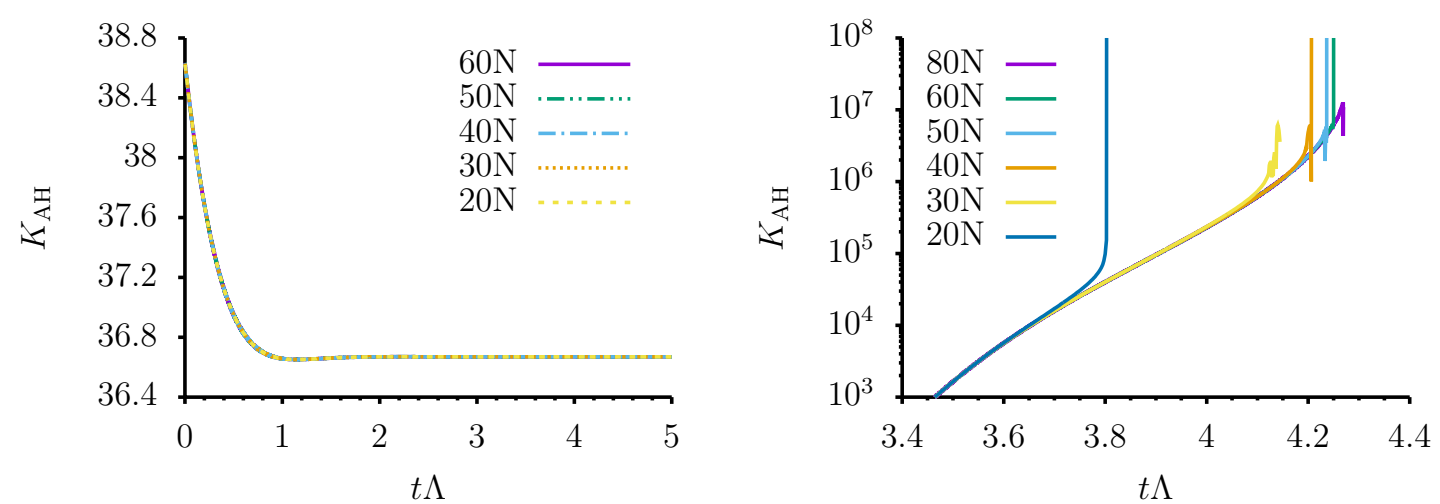

Figure 16. Kretschmann scalar at apparent horizon. Left panel stable case. Right panel unstable case.

both the stable and unstable configurations. For the former case, all resolutions show an excellent agreement. In contrast, the unstable case illustrates a convergence to a divergent behavior which requires increasingly finer resolutions to be captured. Such more finely resolved studies provide enough information to understand the late time behaviour, in particular, up to a time $t \Lambda \simeq 4.2$.

\section{A.5 Event horizon finder}

To find the event horizon we trace null geodesics at late times back in time and determine the surface $R(t)$ where they converge. To do so, we start from

$$
g_{a b} n^{a} n^{b}=0
$$

where $n^{a}$ denotes the null tangent vector to the geodesics. Using (3.1) and the field redefinition (A.1) and (A.2) this relation implies

$$
\frac{\mathrm{d} x}{\mathrm{~d} t}=-x^{2}\left(a(t, x)+\frac{1}{2} \sigma(t, x)^{2}\right)-x \sigma(t, x)-\frac{1}{2},
$$

which we solve numerically using either a RK4 integrator or an second order implicit integrator. The results obtained with both methods converge and are in excellent agreement. As described briefly above, we consider a collection of starting points at different radii and bisect the resulting behavior to home-in on $R(t)$. Figure 17 displays eight representative initial conditions and illustrate the convergent behavior towards the event horizon.

\section{B Bounded scalar potentials in exotic holographic model}

In this section we explore modification of the model (2.1) where the potential for the gravitational scalar $\chi$ is bounded; specifically we modify the nonlinear interactions between $\phi$ and $\chi$ as follows

$$
-g \phi^{2} \chi^{2} \quad \rightarrow \quad-g \phi^{2} \chi^{2}\left(1-f \chi^{2}\right)
$$




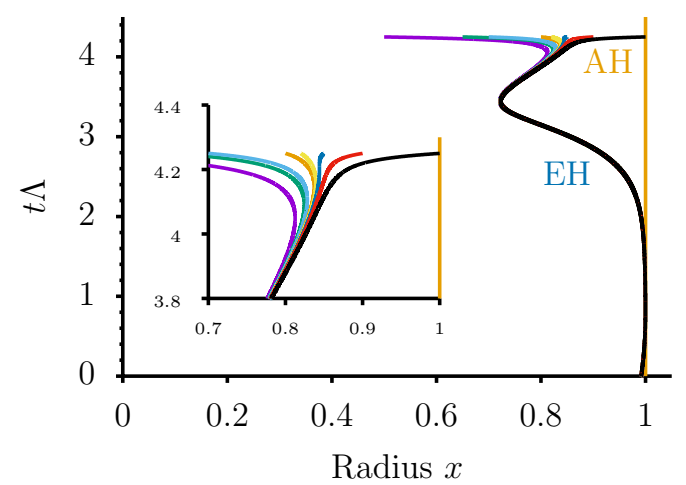

Figure 17. Illustration of representative null rays traced to find the event horizon. The inset shows a zoom-in at late times which aids to visualize how null rays starting at different locations converge as they are traced backwards in time.
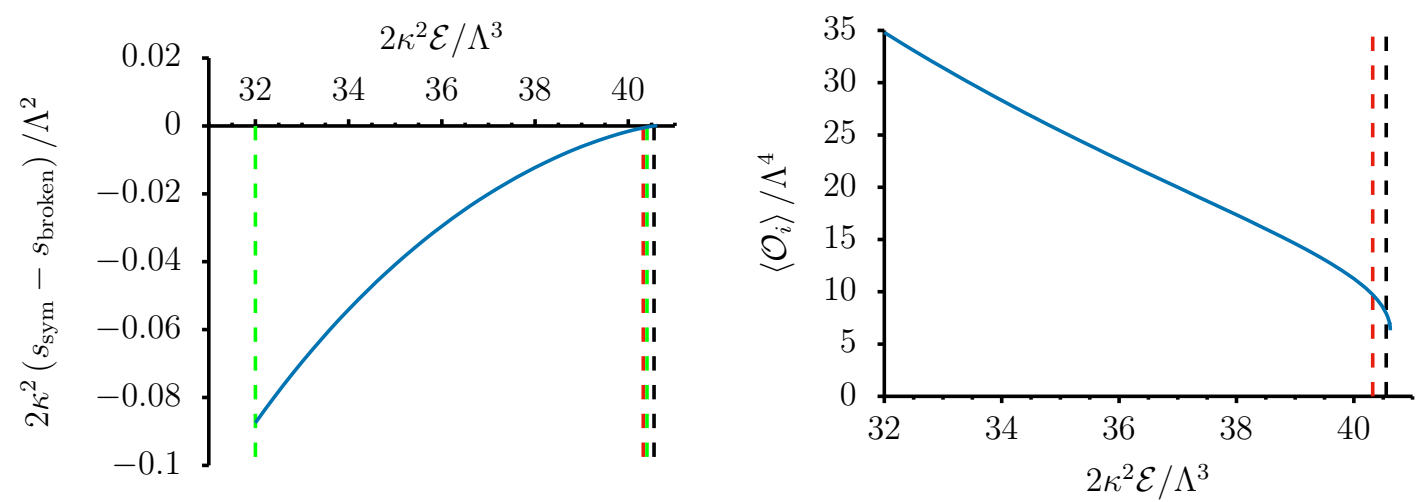

Figure 18. There is a new symmetry broken phase of the model (2.1) for the modified nonlinear interaction (B.1); here, $f=55$. The left panel shows the difference in the entropy densities between symmetric and broken phases as a function of energy density. The (red) vertical dashed line is the onset of the linearized instability of the symmetric phase at $\mathcal{E}=\mathcal{E}_{\text {crit }}$, see (2.5). The (black) vertical dashed line denotes a new first-order phase transition at $\mathcal{E}=\mathcal{E}_{\text {black }}$, see (B.2). The vertical (green) dashed lines indicate energy densities used in numerical evolutions, $\mathcal{E}_{\text {green,left }}<\mathcal{E}_{\text {crit }}<\mathcal{E}_{\text {green,right }}<$ $\mathcal{E}_{\text {black }}$. The right panel represents the order parameter of the broken phase as a function of the energy density.

where $f=$ const $>0$ is a new parameter. It is straightforward to modify the numerical code to reflect the change (B.1). We performed various tests and verified convergence of the new numerical code. In what follows we report the results of the analysis.

Because modification (B.1) is a higher-order $\chi$-nonlinear interaction, the linearized stability analyses are not affected - there is a linearized instability for $\mathcal{E}<\mathcal{E}_{\text {crit }}$ with $\mathcal{E}_{\text {crit }}$ given by (2.5). Likewise, the static exotic branch bifurcating from the symmetric phase at the onset of the instability is qualitatively unchanged, see figure 3. However, for a wide range of $f>0$ we found a new phase of the model with $\left\langle\mathcal{O}_{i}\right\rangle \neq 0$. This new phase exists for $\mathcal{E} \lessgtr \mathcal{E}_{\text {crit }}$, though it is numerically challenging to find it as $f$ decreases and $\mathcal{E} \rightarrow \mathcal{E}_{\text {crit }}$. The new branch enters a full phase diagram of the model in a fairly complicated fashion. 

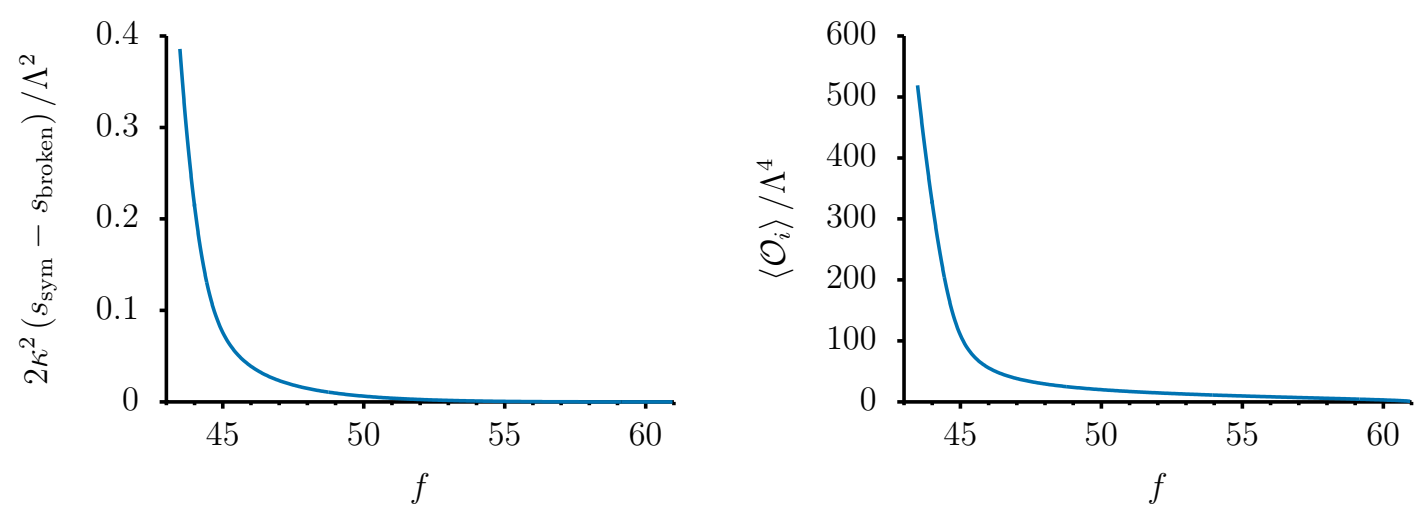

Figure 19. New symmetry broken phase dominates the microcanonical ensemble at $\mathcal{E}=\mathcal{E}_{\text {crit }}$ for a wide range of the nonlinear parameter $f$, "bounding" the scalar potential in (B.1). Right panel shows the corresponding dependence of the order parameter $\left\langle\mathcal{O}_{i}\right\rangle$ in the broken phase.

For the results in figure 18 we choose $f=55$. The left panel shows the entropy density difference between the symmetric phase $s_{\text {sym }}$ and a new symmetry broken phase $s_{\text {broken }}$. The (red) dashed vertical line identifies the onset of the linearized instability at $\mathcal{E}=\mathcal{E}_{\text {crit }}$. This new phase dominates the microcanonical ensemble all the way to $\mathcal{E}_{\text {black }}$, denoted by the (black) vertical dashed line,

$$
s_{\text {broken }}(\mathcal{E})>s_{\text {sym }}(\mathcal{E}), \quad \text { for } \quad \mathcal{E}<\mathcal{E}_{\text {black }}=1.0057(3) \mathcal{E}_{\text {crit }}
$$

At $\mathcal{E}=\mathcal{E}_{\text {black }}$ there is a first-order phase transition, and since the symmetric phase at this energy density is perturbatively stable, the transition would occur dynamically only if the amplitude of the symmetry breaking fluctuations is large enough — we explore this below for the energy density represented by the right (green) vertical dashed line

$$
\mathcal{E}_{\text {crit }} \quad<\quad \mathcal{E}_{\text {green,right }}=1.0019(7) \mathcal{E}_{\text {crit }} \quad<\quad \mathcal{E}_{\text {black }}
$$

The left (green) vertical dashed line corresponds to the energy density (3.18), $\mathcal{E}_{\text {green,left }}<$ $\mathcal{E}_{\text {crit }}$. The right panel in figure 18 shows the order parameter, $\left\langle\mathcal{O}_{i}\right\rangle$, for the new symmetry breaking phase as a function of the energy density $\mathcal{E}$.

As figure 19 shows, the new symmetry broken phase dominates the microcanonical ensemble at $\mathcal{E}=\mathcal{E}_{\text {crit }}$ for all values of the $f$ in (B.1) we studied. Notice that as $f$ decreases the new phase becomes very different from the symmetric phase: it is much strongly favored entropically, and the symmetry breaking order parameter $\left\langle\mathcal{O}_{i}\right\rangle$ (right panel) exhibits a rapid growth. All this is suggests that the limit $f \rightarrow 0_{+}$is a singular one, as expected from the main text analysis of the $f=0$ model (2.1).

Figure 20 represents the time evolution of model with $f=55, \mathcal{E}=\mathcal{E}_{\text {green,left }}=$ $0.793642 \mathcal{E}_{\text {crit }}$, and the initial conditions chosen following appendix A.3 with values of $\mathcal{A}_{p}$ and $\mathcal{A}_{q}$ as in the simulations reported in section 3.4. The symmetry preserving $\left\langle\mathcal{O}_{r}\right\rangle$ (left panel) and the symmetry breaking $\left\langle\mathcal{O}_{i}\right\rangle$ (right panel) condensates equilibrate to static values [(red) dashed lines] corresponding to the new symmetry broken phase discussed here. This should be contrasted with the $f=0$ results reported in section 3.4, where the system 

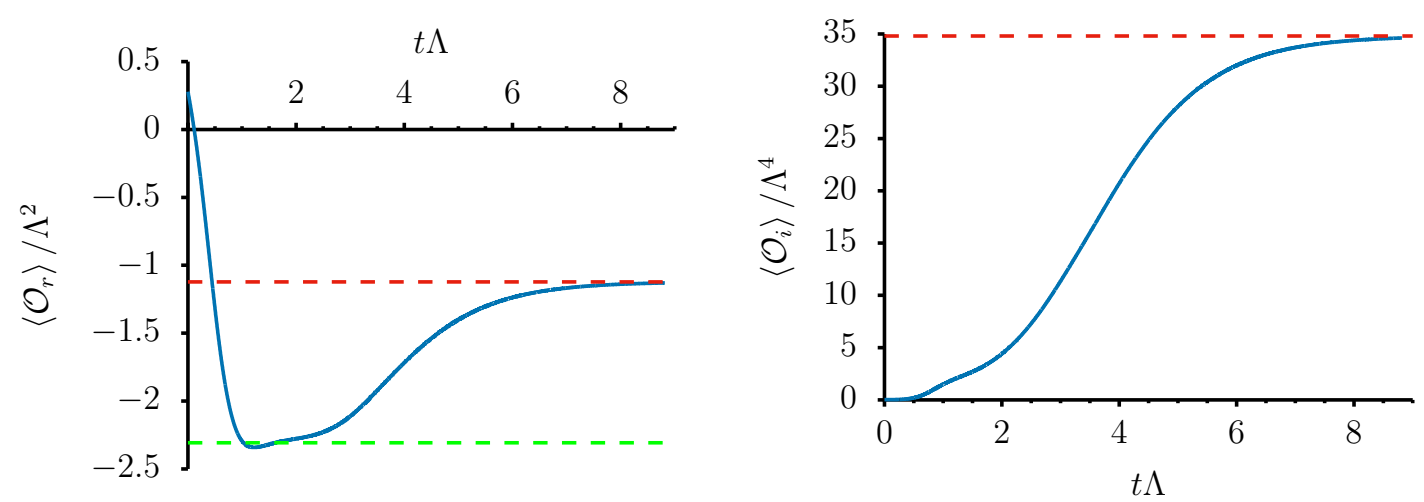

Figure 20. Time evolution of the symmetric preserving order parameter $\left\langle\mathcal{O}_{r}\right\rangle$ (left panel) and the symmetry breaking order parameter $\left\langle\mathcal{O}_{i}\right\rangle$ (right panel) for $\mathcal{E}<\mathcal{E}_{\text {crit }}$, see (3.18), and $f=55$. The system equilibrates to appropriate static values of the condensates, represented by (red) dashed lines. The (green) dashed line is the expectation value of $\mathcal{O}_{r}$ in the symmetric phase at the corresponding energy density.
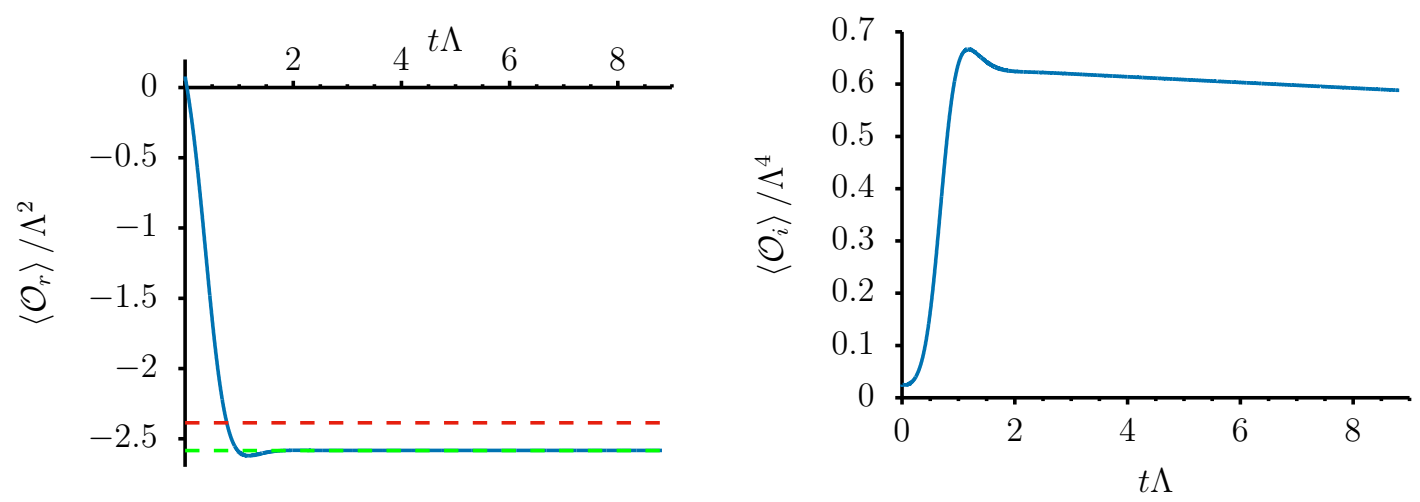

Figure 21. Dynamics of the model with $f=55$ and $\mathcal{E}=\mathcal{E}_{\text {green,right }}$, see (B.3) with initially small amplitude of the symmetry breaking fluctuations (right panel). Symmetry preserving condensate $\left\langle\mathcal{O}_{r}\right\rangle$ equilibrates to the value in the symmetric phase [(green) dashed line]. The (red) dashed line is the value of this condensate at the same energy in the symmetry broken phase.

evolves to a naked singularity. We initiate evolution with small amplitude of the symmetry breaking fluctuation

$$
\left.\left\langle\mathcal{O}_{i}\right\rangle\right|_{t=0}=0.0252 \Lambda^{4} ;
$$

hence, they do not have enough time to become nonlinear at $t \Lambda \sim 1$, and the symmetric condensate $\left\langle\mathcal{O}_{r}\right\rangle$ is close to its value in symmetry preserving phase at the corresponding energy density (represented by (green) dashed line). For $t \Lambda>1$ the symmetry breaking fluctuations continue to grow, ultimately capping off at the new equilibrium value.

As shown in figure 18, the new symmetry broken phase has an interesting feature in the narrow energy range:

$$
\mathcal{E}_{\text {crit }}<\mathcal{E}<\mathcal{E}_{\text {black }}
$$



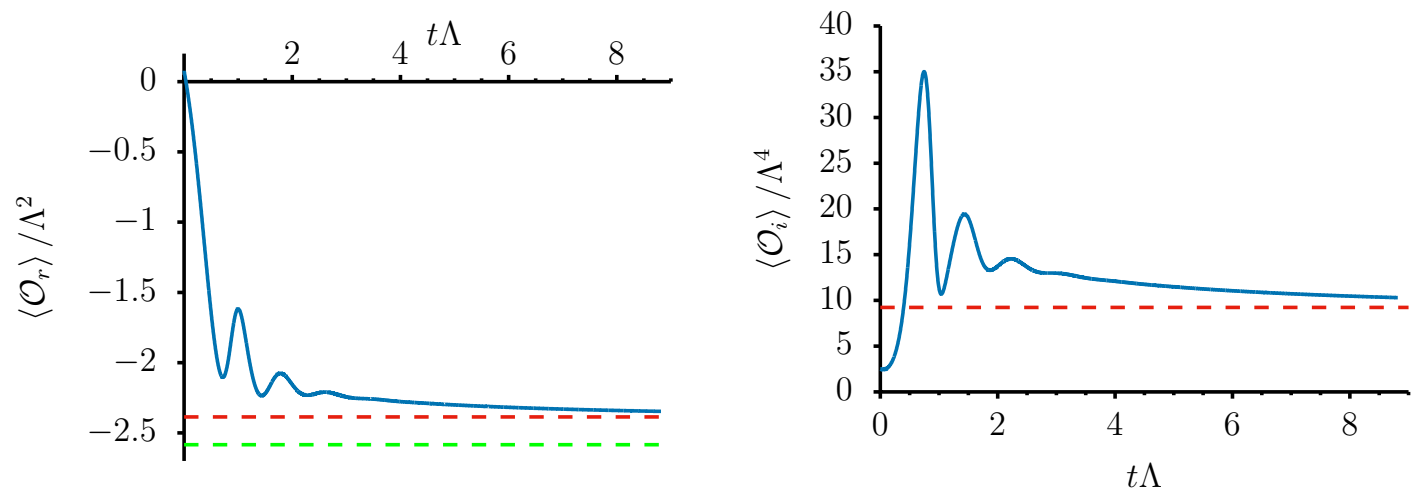

Figure 22. Dynamics of the model with $f=55$ and $\mathcal{E}=\mathcal{E}_{\text {green,right }}$, see (B.3) with initially large amplitude of the symmetry breaking fluctuations (right panel). Symmetry preserving condensate $\left\langle\mathcal{O}_{r}\right\rangle$ approaches the equilibrium value in the symmetry broken phase [ (red) dashed line]. The (green) dashed line is the value of this condensate at the same energy in the symmetric phase.

Here, the symmetric phase is perturbatively stable, but the new symmetry broken phase is nonetheless entropically favorable; thus one excepts that the broken phase can be reached dynamically only if the amplitude of the initial symmetry breaking fluctuations is sufficiently large. We find that this is indeed the case. For the results presented in figure $21,\left.\left\langle\mathcal{O}_{i}\right\rangle\right|_{t=0}=0.0252 \Lambda^{4}$ and the system equilibrates to a (metastable) symmetric phase. Figure 22 represents results of the simulation for the initial condition with $\left.\left\langle\mathcal{O}_{i}\right\rangle\right|_{t=0}=2.52 \Lambda^{4}$ - here the amplitude is large enough to reach the entropically dominant symmetry broken phase. The approach to equilibrium in both cases is very slow as the energy density of the simulations is close to the critical one, see (B.3).

Open Access. This article is distributed under the terms of the Creative Commons Attribution License (CC-BY 4.0), which permits any use, distribution and reproduction in any medium, provided the original author(s) and source are credited.

\section{References}

[1] J.M. Maldacena, The large- $N$ limit of superconformal field theories and supergravity, Int. J. Theor. Phys. 38 (1999) 1113 [Adv. Theor. Math. Phys. 2 (1998) 231] [hep-th/9711200] [INSPIRE].

[2] O. Aharony, S.S. Gubser, J.M. Maldacena, H. Ooguri and Y. Oz, Large-N field theories, string theory and gravity, Phys. Rept. 323 (2000) 183 [hep-th/9905111] [INSPIRE].

[3] S.A. Hartnoll, C.P. Herzog and G.T. Horowitz, Building a holographic superconductor, Phys. Rev. Lett. 101 (2008) 031601 [arXiv:0803.3295] [INSPIRE].

[4] S.A. Hartnoll, C.P. Herzog and G.T. Horowitz, Holographic superconductors, JHEP 12 (2008) 015 [arXiv:0810.1563] [INSPIRE].

[5] I.R. Klebanov and E. Witten, AdS/CFT correspondence and symmetry breaking, Nucl. Phys. B 556 (1999) 89 [hep-th/9905104] [INSPIRE]. 
[6] R. Gregory and R. Laflamme, Black strings and p-branes are unstable, Phys. Rev. Lett. 70 (1993) 2837 [hep-th/9301052] [INSPIRE].

[7] S.A. Hartnoll, Lectures on holographic methods for condensed matter physics, Class. Quant. Grav. 26 (2009) 224002 [arXiv:0903.3246] [INSPIRE].

[8] A. Buchel and C. Pagnutti, Exotic hairy black holes, Nucl. Phys. B 824 (2010) 85 [arXiv: 0904.1716] [INSPIRE].

[9] A. Donos and J.P. Gauntlett, Superfluid black branes in $A d S_{4} \times S^{7}$, JHEP 06 (2011) 053 [arXiv:1104.4478] [INSPIRE].

[10] V.E. Hubeny and M. Rangamani, Unstable horizons, JHEP 05 (2002) 027 [hep-th/0202189] [INSPIRE].

[11] A. Buchel and L. Lehner, Small black holes in $A d S_{5} \times S^{5}$, Class. Quant. Grav. 32 (2015) 145003 [arXiv: 1502.01574] [INSPIRE].

[12] A. Buchel, Universality of small black hole instability in AdS/CFT, arXiv:1509.07780 [INSPIRE].

[13] O.J.C. Dias, J.E. Santos and B. Way, Lumpy $A d S_{5} \times S^{5}$ black holes and black belts, JHEP 04 (2015) 060 [arXiv: 1501.06574] [INSPIRE].

[14] P.M. Chesler and L.G. Yaffe, Numerical solution of gravitational dynamics in asymptotically anti-de Sitter spacetimes, JHEP 07 (2014) 086 [arXiv:1309.1439] [INSPIRE].

[15] S.S. Gubser, Phase transitions near black hole horizons, Class. Quant. Grav. 22 (2005) 5121 [hep-th/0505189] [INSPIRE].

[16] A. Buchel and C. Pagnutti, Correlated stability conjecture revisited, Phys. Lett. B 697 (2011) 168 [arXiv: 1010.5748] [INSPIRE].

[17] S.S. Gubser and I. Mitra, Instability of charged black holes in Anti-de Sitter space, hep-th/0009126 [INSPIRE].

[18] S.S. Gubser and I. Mitra, The evolution of unstable black holes in anti-de Sitter space, JHEP 08 (2001) 018 [hep-th/0011127] [INSPIRE].

[19] H. Bondi, M.G.J. van der Burg and A.W.K. Metzner, Gravitational waves in general relativity. VII. Waves from axi-symmetric isolated systems, Proc. Roy. Soc. London Ser. A 269 (1962) 21.

[20] N.T. Bishop, R. Gomez, L. Lehner, M. Maharaj and J. Winicour, High powered gravitational news, Phys. Rev. D 56 (1997) 6298 [gr-qc/9708065] [InSPIRE].

[21] J. Winicour, Characteristic evolution and matching, arXiv:0810.1903 [INSPIRE].

[22] I. Booth, Black hole boundaries, Can. J. Phys. 83 (2005) 1073 [gr-qc/0508107] [InSPIRE].

[23] P. Figueras, V.E. Hubeny, M. Rangamani and S.F. Ross, Dynamical black holes and expanding plasmas, JHEP 04 (2009) 137 [arXiv: 0902.4696] [INSPIRE].

[24] A. Buchel, Chiral symmetry breaking in cascading gauge theory plasma, Nucl. Phys. B 847 (2011) 297 [arXiv: 1012.2404] [INSPIRE].

[25] P. Bosch, S.R. Green and L. Lehner, Nonlinear evolution and final fate of charged Anti-de Sitter black hole superradiant instability, Phys. Rev. Lett. 116 (2016) 141102 [arXiv: 1601.01384] [INSPIRE]. 
[26] T. Crisford and J.E. Santos, Violating the weak cosmic censorship conjecture in four-dimensional Anti-de Sitter space, Phys. Rev. Lett. 118 (2017) 181101 [arXiv: 1702.05490] [INSPIRE].

[27] A. Buchel, P.M. Chesler and L.G. Yaffe, in progress.

[28] M. Srednicki, Chaos and quantum thermalization, Phys. Rev. E 50 (1994) 888 [cond-mat/9403051].

[29] P. Bizon and A. Rostworowski, On weakly turbulent instability of Anti-de Sitter space, Phys. Rev. Lett. 107 (2011) 031102 [arXiv:1104.3702] [INSPIRE].

[30] A. Buchel, L. Lehner and S.L. Liebling, Scalar collapse in AdS, Phys. Rev. D 86 (2012) 123011 [arXiv: 1210.0890] [INSPIRE].

[31] A. Buchel, S.L. Liebling and L. Lehner, Boson stars in AdS spacetime, Phys. Rev. D 87 (2013) 123006 [arXiv:1304.4166] [INSPIRE].

[32] V. Balasubramanian, A. Buchel, S.R. Green, L. Lehner and S.L. Liebling, Holographic thermalization, stability of Anti-de Sitter space and the Fermi-Pasta-Ulam paradox, Phys. Rev. Lett. 113 (2014) 071601 [arXiv:1403.6471] [INSPIRE].

[33] S.R. Green, A. Maillard, L. Lehner and S.L. Liebling, Islands of stability and recurrence times in AdS, Phys. Rev. D 92 (2015) 084001 [arXiv:1507.08261] [INSPIRE].

[34] O.J. Dias, J.E. Santos and B. Way, Localised $A d S_{5} \times S^{5}$ black holes, Phys. Rev. Lett. 117 (2016) 151101 [arXiv: 1605.04911] [INSPIRE].

[35] B. Withers, Black branes dual to striped phases, Class. Quant. Grav. 30 (2013) 155025 [arXiv: 1304.0129] [INSPIRE].

[36] K. Pilch and N.P. Warner, $N=2$ supersymmetric RG flows and the IIB dilaton, Nucl. Phys. B 594 (2001) 209 [hep-th/0004063] [INSPIRE].

[37] A. Buchel, A.W. Peet and J. Polchinski, Gauge dual and noncommutative extension of an $N=2$ supergravity solution, Phys. Rev. D 63 (2001) 044009 [hep-th/0008076] [INSPIRE].

[38] A. Buchel, J.G. Russo and K. Zarembo, Rigorous test of non-conformal holography: Wilson loops in $N=2 *$ theory, JHEP 03 (2013) 062 [arXiv: 1301.1597] [INSPIRE].

[39] D. Garfinkle, Numerical simulations of generic singuarities, Phys. Rev. Lett. 93 (2004) 161101 [gr-qc/0312117] [INSPIRE].

[40] A. Buchel, R.C. Myers and A. van Niekerk, Nonlocal probes of thermalization in holographic quenches with spectral methods, JHEP 02 (2015) 017 [Erratum ibid. 07 (2015) 137] [arXiv: 1410.6201] [INSPIRE]. 